\title{
WATER RESOURCES OF THE RIVER RAISIN BASIN SOUTHEASTERN MICHIGAN
}

\author{
By
}

\author{
R. L. Knutilla and W. B. Allen
}

\section{PHYSIOGRAPHIC DATA FOR SELECTED SUBBASINS IN THE RIVER RAISIN BASIN}

The accompanying tables summarize some physiographic data for selected subbasins in the River Raisin basin having about 2 or more square miles of drainage area. Selected subbasins of the major streams include area above mouths of tributaries, and points of interest such as highways, towns, gaging stations, or dams, and below the confluence of the main stream and a tributary so as to include both drainage areas. Similar criteria were used for tributary streams, except where the tributary was too small to merit further dividing. Most subbasins are shown on the basin map, sheet 1 . To help locate the tabulated areas on the map, each subbasin is assigned a number and numbers for selected subbasins are shown on the map at the downstream end of the subbasins. Also shown are names of main roads and river miles, at 5-mile intervals, for the River Raisin and two of its main tributaries.

Basin divides were outlined on the latest $7 \frac{1}{2}-$ or 15 minute topographic maps, scale $1: 24,000$ or $1: 62,500$, respectively, and drainage areas determined in accordance with the standards set forth by the Committee on Hydrology, Water Resources Council (formerly the Subcommittee on Hydrology, Inter-Agency Committee on Water Resources). Similarly, river miles were determined in accordance with the Committee's standards. Mile zero is considered to be the mouth of the stream. The source of a stream is considered to be the upper end of the stream, whether or not it is perennial. A defined channel which may be inferred from topographic maps to carry water occasionally is, therefore, inclured in the stream length. A stream that has its beginning at the confluence of two tributaries is considere to be a continuation of the longer tributary and to have as its source the source of that tributary. Its average slope is determined by treating the st:-eam and tributary as one. Beaver Creek (number 253), for example, is considered to be the contin'ation of Slater Creek. In the table Slater Creek has been parenthetically noted as being the head"waters of Beaver Creek. Other tributary streams, in ti is classification, are likewise noted.

Altitudes shown for site and source are f-om topographic maps and represent the altitude of the water surface at approximately normal river stage, the datum being mean sea level. Average slopes were computed from the mileage between and differences in altitude of site and source and represent the fall of the stream in feet per mile.

Streams are listed in a downstream order along the main stem, with tributary streams entered above the next main-stem location. A similar order is followed for listing streams of first rank, second rank, or other rank. To indicate the rank, and the stream to which it is tributary, the stream name is inderted, each indentation representing one rank. The st:-am rank is further identified by numbers in th: column heading with stream name and location. Number 1 applies to River Raisin. Principal tributaries are under number 2 , their tributaries under number 3 , and so on.

PHYSIOGRAPHIC DATA FOR SELECTED SUBBASINS IN THE RIVER RAISIN BASIN

\begin{tabular}{|c|c|c|c|c|c|c|c|}
\hline \multirow{2}{*}{$\begin{array}{l}\text { Num- } \\
\text { ber } \\
\text { key }\end{array}$} & Stream and location & \multirow{2}{*}{$\begin{array}{c}\text { Drainage } \\
\text { area } \\
\text { (sq } \mathrm{mi} \text { ) }\end{array}$} & \multicolumn{2}{|c|}{ Miles } & \multicolumn{2}{|c|}{$\begin{array}{c}\text { Altitude } \\
\text { (ft above msl) }\end{array}$} & \multirow{2}{*}{$\begin{array}{l}\text { Average } \\
\text { slope } \\
\text { (ft per } \\
\text { mile) }\end{array}$} \\
\hline & 123456 Ranking order & & $\begin{array}{l}\text { above } \\
\text { mouth }\end{array}$ & $\begin{array}{l}\text { below } \\
\text { source }\end{array}$ & $\begin{array}{l}\text { at } \\
\text { site }\end{array}$ & $\begin{array}{c}\text { at } \\
\text { source }\end{array}$ & \\
\hline & \multirow{2}{*}{$\begin{array}{c}\text { River Raisin above Onemile Lake outlet, } \\
\text { NW1/4 sec. } 6 \text {, T. } 5 \text { S., R. } 2 \text { E. } \\
\text { Onemile Lake outlet above Grassy Lake outlet, } \\
\text { SW } 1 / 4 \text { sec. } 12 \text {, T. } 5 \text { S., R. } 1 \text { E. }\end{array}$} & 3.95 & 128.8 & 4.9 & 978 & 1,030 & 10.6 \\
\hline 2. & & 7.17 & 2.3 & 4.0 & 985 & 1,025 & 10.0 \\
\hline 3. & \multirow{2}{*}{$\begin{array}{l}\text { Grassy Lake outlet at mouth, } \\
\text { SW1/4 sec. 12, T. } 5 \text { S., R. } 1 \text { E. } \\
\text { Onemile Lake outlet at Grassy Lake outlet, } \\
\text { SW } 1 / 4 \text { sec. 12, T. } 5 \text { S., R. } 1 \text { E. }\end{array}$} & 3.57 & 0 & 3.5 & 975 & 998 & 6.6 \\
\hline 4. & & 10.7 & 2.3 & 4.0 & 975 & 1,025 & 10.0 \\
\hline
\end{tabular}


PHYSIOGRAPHIC DATA FOR SELECTED SUBBASINS IN THE RIVER RAISIN BASIN - Continued

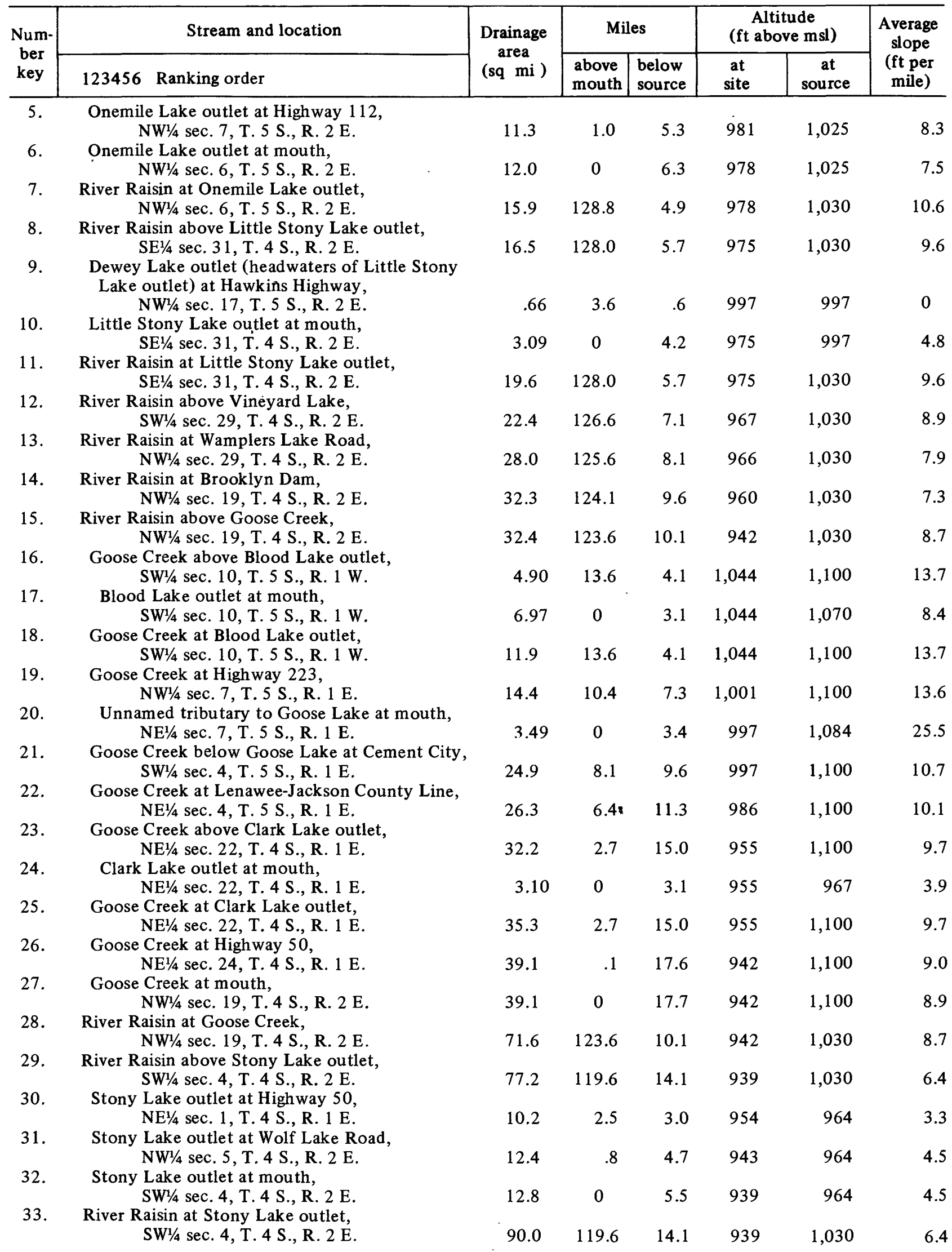


PHYSIOGRAPHIC DATA FOR SELECTED SUBBASINS IN THE RIVER RAISIN BASIN - Continu?d

\begin{tabular}{|c|c|c|c|c|c|c|c|}
\hline \multirow{2}{*}{$\begin{array}{c}\text { Num- } \\
\text { ber } \\
\text { key }\end{array}$} & Stream and location & \multirow{2}{*}{$\begin{array}{l}\text { Drainage } \\
\text { area } \\
\text { (sq mi ) }\end{array}$} & \multicolumn{2}{|c|}{ Miles } & \multicolumn{2}{|c|}{$\begin{array}{c}\text { Altitude } \\
\text { (ft above msl) }\end{array}$} & \multirow{2}{*}{$\begin{array}{c}\text { Average } \\
\text { slope } \\
\text { (ft per } \\
\text { mile) } \\
\end{array}$} \\
\hline & 123456 Ranking order & & $\begin{array}{l}\text { above } \\
\text { mouth }\end{array}$ & $\begin{array}{l}\text { below } \\
\text { source }\end{array}$ & $\begin{array}{c}\text { at } \\
\text { site }\end{array}$ & $\begin{array}{c}\text { at } \\
\text { source }\end{array}$ & \\
\hline 34. & $\begin{array}{l}\text { River Raisin at Norvell Dam, } \\
\text { NW11/4 sec. 3, T. } 4 \text { S., R. } 2 \text { E. }\end{array}$ & 92.4 & 118.2 & 15.5 & 939 & 1,030 & 5.8 \\
\hline 35. & $\begin{array}{l}\text { River Raisin above Bessey Lake outlet, } \\
\text { NE1/4 sec. 3, T. } 4 \text { S., R. } 2 \text { E. }\end{array}$ & 93.0 & 117.7 & 16.0 & 930 & 1,030 & 6.3 \\
\hline 36. & $\begin{array}{l}\text { Bessy Lake outlet above unnamed tributary, } \\
\text { SW1/4 sec. } 22 \text {, T. } 3 \text { S., R. } 2 \text { E. }\end{array}$ & 1.77 & 3.7 & 1.0 & 951 & 960 & 9.0 \\
\hline 37 & $\begin{array}{l}\text { Unnamed tributary at mouth, } \\
\text { SW } 1 / 4 \text { sec. } 22 \text {, T. } 3 \text { S., R. } 2 \text { E. }\end{array}$ & 6.60 & 0 & 3.1 & 951 & 987 & 11.6 \\
\hline 38. & $\begin{array}{l}\text { Bessy Lake outlet at unnamed tributary, } \\
\text { SW1/4 sec. } 22 \text {, T. } 3 \text { S., R. } 2 \text { E. } \\
\text { Bessy Lake outlet above Swezzey Lake outlet, }\end{array}$ & 8.37 & 3.7 & 1.0 & 951 & 960 & 9.0 \\
\hline 39. & $\begin{array}{l}\text { Bessy Lake outlet above Swezzey Lake outlet, } \\
\mathrm{NE}^{1 / 4} \mathrm{sec} .3, \mathrm{~T} .4 \mathrm{~S} ., \mathrm{R} .2 \mathrm{E} \text {. }\end{array}$ & 11.2 & .2 & 4.5 & 930 & 960 & 6.7 \\
\hline 40. & $\begin{array}{l}\text { Swezzey Lake outlet at mouth, } \\
\text { NE1/4 sec. } 3, \text { T. } 4 \text { S., R. } 2 \text { E. }\end{array}$ & 2.34 & 0 & .8 & 930 & 930 & 0 \\
\hline 41. & $\begin{array}{l}\text { Bessy Lake outlet at Swezzy Lake outlet, } \\
\text { NE1/4 sec. 3, T. } 4 \text { S., R. } 2 \text { E. }\end{array}$ & 13.5 & .2 & 4.5 & 930 & 960 & 6.7 \\
\hline 42. & $\begin{array}{l}\text { Bessy Lake outlet at mouth, } \\
\text { NE1/4 sec. } 3, \text { T. } 4 \text { S., R. } 2 \text { E. }\end{array}$ & 13.6 & 0 & 4.7 & 930 & 960 & 6.4 \\
\hline 43. & $\begin{array}{l}\text { River Raisin at Bessy Lake outlet, } \\
\text { NE1/4 sec. 3, T. } 4 \text { S., R. } 2 \text { E. }\end{array}$ & 107 & 117.7 & 16.0 & 930 & 1,030 & 6.3 \\
\hline $\begin{array}{l}44 . \\
45 .\end{array}$ & $\begin{array}{l}\text { River Raisin above Fay Lake outlet, } \\
\text { NW1/4 sec. } 6, \text { T. } 4 \text { S., R. } 3 \mathrm{E} \text {. } \\
\text { Fay Lake outlet at mouth, }\end{array}$ & 110 & 114.7 & 19.0 & 916 & 1,030 & 6.0 \\
\hline 46. & $\begin{array}{l}\text { NW1/4 sec. } 6, \text { T. } 4 \text { S., R. } 3 \text { E. } \\
\text { River Raisin at Fay Lake outlet, }\end{array}$ & 9.19 & 0 & 5.6 & 916 & 950 & 6.1 \\
\hline 47. & $\begin{array}{c}\mathrm{NW} 1 / 4 \text { sec. } 6, \mathrm{~T} .4 \mathrm{~S} ., \mathrm{R} .3 \mathrm{E} . \\
\text { River Raisin above unnamed tributary, }\end{array}$ & 120 & 114.7 & 19.0 & 916 & 1,030 & 6.0 \\
\hline 48. & $\begin{array}{l}\text { SW1/4 sec. } 29, \text { T. } 3 \mathrm{~S} ., \mathrm{R} .3 \mathrm{E} \text {. } \\
\text { Unnamed tributary at mouth, }\end{array}$ & 122 & 111.6 & 22.1 & 906 & 1,030 & 5.6 \\
\hline 49. & $\begin{array}{l}\text { SW11/4 sec. } 29, \text { T. } 3 \text { S., R. } 3 \text { E. } \\
\text { River Raisin at unnamed tributary. }\end{array}$ & 6.14 & 0 & 2.4 & 906 & 1,005 & 41.3 \\
\hline & SE $1 / 4$ sec. 29 , T. 3 S., R. 3 E. & 129 & 110.8 & 22.9 & 904 & 1,030 & 5.5 \\
\hline 51. & $\begin{array}{l}\text { River Raisin at Sharon Valley Road (gage), } \\
\text { SE1/4 sec. 33, T. } 3 \text { S., R. } 3 \mathrm{E} \text {. }\end{array}$ & 132 & 109.3 & 24.4 & 899 & 1,030 & 5.4 \\
\hline $\begin{array}{l}52 . \\
53 .\end{array}$ & $\begin{array}{l}\text { River Raisin above unnamed tributary, } \\
\text { NW1/4 sec. } 3, \text { T. } 4 \text { S., R. } 3 \mathrm{E} \text {. } \\
\text { Unnamed tributary at mouth, }\end{array}$ & 134 & 108.2 & 25.5 & 892 & 1,030 & 5.4 \\
\hline & NW1/4 sec. 3 , T. 4 S., R. 3 E. & 5.92 & 0 & 2.7 & 892 & 965 & 27.0 \\
\hline 54. & $\begin{array}{l}\text { River Raisin at unnamed tributary, } \\
\text { NW1/4 sec. } 3, \text { T. } 4 \text { N., R. } 3 \text { E. }\end{array}$ & 140 & 108.2 & 25.5 & 892 & 1,030 & 5.4 \\
\hline 55. & $\begin{array}{l}\text { River Raisin at Manchester Dam, Austin Road, } \\
\text { SE } 1 / 4 \text { sec. 2, T. } 4 \text { S., R. } 3 \text { E. } \\
\text { River Raisin at Ford Dam }\end{array}$ & 142 & 106.4 & 27.3 & 880 & $1,03 n$ & 5.5 \\
\hline $\begin{array}{l}56 . \\
57 .\end{array}$ & $\begin{array}{l}\text { River Raisin at Ford Dam, Austin Road, } \\
\text { SW1/4 sec. } 1 \text {, T. } 4 \text { S., R. } 3 \text { E. } \\
\text { River Raisin above unnamed tributary, }\end{array}$ & 148 & 105.1 & 28.6 & 860 & $1,03 n$ & 5.9 \\
\hline 58. & $\begin{array}{l}\mathrm{SE} 1 / 4 \mathrm{sec} .7, \mathrm{~T} .4 \mathrm{~S} ., \mathrm{R} .4 \mathrm{E} \text {. } \\
\text { Unnamed tributary at mouth, }\end{array}$ & 150 & 102.4 & 31.3 & 838 & 1,030 & 6.1 \\
\hline & $\begin{array}{l}\text { SE } 1 / 4 \text { sec. } 7, \text { T. } 4 \text { S., R. } 4 \text { E. } \\
\text { River Raisin at unnamed tributary, }\end{array}$ & 2.89 & 0 & 2.2 & 838 & 940 & 46.4 \\
\hline & $\begin{array}{l}\text { River Raisin at unnamed tributary, } \\
\mathrm{SE}^{1 / 4} \text { sec. } 7 \text {, T. } 4 \text { S., R. } 4 \mathrm{E} \text {. }\end{array}$ & 153 & 102.4 & 31.3 & 838 & $1,03 ?$ & 6.1 \\
\hline $\begin{array}{l}60 . \\
61 .\end{array}$ & $\begin{array}{l}\text { River Raisin above unnamed tributary, } \\
\text { SW } 1 / 4 \mathrm{sec} .8, \mathrm{~T} .4 \mathrm{~S} ., \mathrm{R} .4 \mathrm{E} \text {. } \\
\text { Unnamed tributary at mouth, }\end{array}$ & 154 & 101.7 & 32.0 & 835 & $1,03 ?$ & 6.1 \\
\hline & $\begin{array}{l}\text { Unnamed tributary at mouth, } \\
\text { SW1/4 sec. } 8, \mathrm{~T} .4 \mathrm{~S} ., \mathrm{R} .4 \mathrm{E} \text {. } \\
\text { River Raisin at unnamed tributary, }\end{array}$ & 5.54 & 0 & 4.2 & 835 & 945 & 26.2 \\
\hline & $\begin{array}{l}\text { SE1/4 sec. } 29 \text {, T. } 4 \text { S., R. } 4 \text { E. } \\
\text { Siver Kalsin at }\end{array}$ & 167 & 96.4 & 37.3 & ,814 & 1,030 & 5.8 \\
\hline
\end{tabular}


PHYSIOGRAPHIC DATA FOR SELECTED SUBBASINS IN THE RIVER RAISIN BASIN -Continued

\begin{tabular}{|c|c|c|c|c|c|c|c|}
\hline \multirow{2}{*}{$\begin{array}{c}\text { Num- } \\
\text { ber } \\
\text { key }\end{array}$} & Stream and location & \multirow{2}{*}{$\begin{array}{l}\text { Drainage } \\
\text { area } \\
\text { (sq } \mathrm{mi} \text { ) }\end{array}$} & \multicolumn{2}{|c|}{ Miles } & \multicolumn{2}{|c|}{$\begin{array}{c}\text { Altitude } \\
\text { (ft above msl) }\end{array}$} & \multirow{2}{*}{$\begin{array}{c}\text { Average } \\
\text { slope } \\
\text { (ft per } \\
\text { mile) }\end{array}$} \\
\hline & 123456 Ranking order & & $\begin{array}{l}\text { above } \\
\text { mouth }\end{array}$ & $\begin{array}{l}\text { below } \\
\text { source }\end{array}$ & $\begin{array}{c}\text { at } \\
\text { site }\end{array}$ & $\begin{array}{c}\text { at } \\
\text { source }\end{array}$ & \\
\hline 65. & $\begin{array}{l}\text { River Raisin above Iron Creek, } \\
\text { SW1/4 sec. 32, T. } 4 \text { S., R. } 4 \text { E. } \\
\text { Round Lake outlet (head waters of Iron Creek) }\end{array}$ & 167 & 95.7 & 38.0 & 812 & 1,030 & 5.7 \\
\hline 66. & $\begin{array}{l}\text { at Wamplers Lake Road, } \\
\text { SE1/4 sec. 36, T. } 4 \text { S., R. } 2 \text { E. } \\
\text { Wamplers Lake outlet at Wamplers Lake Road, }\end{array}$ & .90 & 13.5 & .5 & 967 & 967 & 0 \\
\hline 67. & $\begin{array}{l}\text { SW1/4 sec. 36, T. } 4 \mathrm{~S} ., \mathrm{R} .2 \mathrm{E} \text {. } \\
\text { Iron Creek at Jackson-Washtenaw County Line, }\end{array}$ & 7.28 & 12.5 & 1.5 & 967 & 967 & 0 \\
\hline 68. & $\begin{array}{l}\mathrm{SE}^{1 / 4} \mathrm{sec} .25, \mathrm{~T} .4 \mathrm{~S} ., \mathrm{R} .2 \mathrm{E} \text {. } \\
\text { Iron Creek at Iron Mill Pond Dam, Sharon Hollow } \\
\text { Road, }\end{array}$ & 10.5 & 10.9 & 3.1 & 960 & 967 & 2.3 \\
\hline 69. & $\begin{array}{l}\text { NW1/4 sec. } 28, \text { T. } 4 \text { S., R. } 3 \text { E. } \\
\text { Iron Creek at Henzie Road, }\end{array}$ & 16.2 & 7.2 & 6.8 & 915 & 967 & 7.6 \\
\hline 70 . & $\begin{array}{l}\mathrm{NE}^{1 / 4} \text { sec. } 27, \text { T. } 4 \text { S., R. } 3 \text { E. } \\
\text { Iron Creek at Bartlet Road, }\end{array}$ & 24.7 & 4.8 & 9.2 & 886 & 967 & 8.8 \\
\hline 71. & $\begin{array}{l}\mathrm{SE}^{1 / 4} \mathrm{sec} .30, \mathrm{~T} .4 \mathrm{~S} ., \mathrm{R} .4 \mathrm{E} . \\
\text { Iron Creek at mouth, }\end{array}$ & 28.8 & .8 & 13.2 & 820 & 967 & 11.1 \\
\hline 72 . & $\begin{array}{l}\text { SW1/4 sec. } 32 \text {, T. } 4 \text { S., R. } 4 \text { E. } \\
\text { River Raisin at Iron Creek, }\end{array}$ & 29.4 & 0 & 14.0 & 812 & 967 & 11.1 \\
\hline 73. & $\begin{array}{l}\mathrm{SW} \mathrm{W}^{1 / 4} \mathrm{sec} .32 \text {, T. } 4 \mathrm{~S} ., \text { R. } 4 \mathrm{E} \text {. } \\
\text { River Raisin at Clinton Dam, }\end{array}$ & 197 & 95.7 & 38.0 & 812 & 1,030 & 5.7 \\
\hline 74. & $\begin{array}{l}\mathrm{NW} 1 / 4 \mathrm{sec} .5, \text { T. } 5 \text { S., R. } 4 \text { E. } \\
\text { River Raisin at Highway } 12 \text {, }\end{array}$ & 200 & 95.0 & 38.7 & 812 & 1,030 & 5.6 \\
\hline 75. & $\begin{array}{l}\text { center sec. } 5 \text {, T. } 5 \text { S., R. } 4 \text { E. } \\
\text { River Raisin above unnamed tributary, }\end{array}$ & 201 & 94.6 & 39.1 & 797 & 1,030 & 6.0 \\
\hline 76. & $\begin{array}{l}\mathrm{SW} 1 / 4 \text { sec. } 5, \mathrm{~T} .5 \mathrm{~S} ., \mathrm{R} .4 \mathrm{E} \text {. } \\
\text { Unnamed tributary at mouth, }\end{array}$ & 202 & 94.3 & 39.4 & 795 & 1,030 & 6.0 \\
\hline 77. & $\begin{array}{l}\text { SW1/4 sec. 5, T. } 5 \text { S., R. } 4 \text { E. } \\
\text { River Raisin at unnamed tributary. }\end{array}$ & 4.46 & 0 & 3.3 & 795 & 883 & 26.7 \\
\hline 78. & $\begin{array}{l}\mathrm{SW} 1 / 4 \mathrm{sec} .5, \mathrm{~T} .5 \mathrm{~S} ., \mathrm{R} .4 \mathrm{E} \text {. } \\
\text { River Raisin at Staib Road, }\end{array}$ & 206 & 94.3 & 39.4 & 795 & 1,030 & $6: 0$ \\
\hline 79. & $\begin{array}{l}\mathrm{NE} 1 / 4 \text { sec. } 17, \text { T. } 5 \text { S., R. } 4 \text { E. } \\
\text { River Raisin above unnamed tributary, }\end{array}$ & 213 & 91.9 & 41.8 & 784 & 1,030 & 5.9 \\
\hline 80. & $\begin{array}{l}\mathrm{NW} 1 / 4 \text { sec. } 21, \mathrm{~T} .5 \mathrm{~S} ., \mathrm{R} .4 \mathrm{E} \text {. } \\
\text { Unnamed tributary at mouth, }\end{array}$ & 213 & 90.8 & 42.9 & 780 & 1,030 & 5.8 \\
\hline 81. & $\begin{array}{l}\mathrm{NW}^{1 / 4} \mathrm{sec} .21, \mathrm{~T} .5 \mathrm{~S} ., \mathrm{R} .4 \mathrm{E} \text {. } \\
\text { River Raisin at unnamed tributary, }\end{array}$ & 1.07 & 0 & 1.4 & 780 & 828 & 34.3 \\
\hline 82. & $\begin{array}{l}\quad \mathrm{NW}^{1 / 4} / 4 \mathrm{sec} .21, \text { T. } 5 \text { S., R. } 4 \text { E. } \\
\text { River Raisin at Penn Central Railroad, }\end{array}$ & 215 & 90.8 & 42.9 & 780 & 1,030 & 5.8 \\
\hline 83. & & 216 & 89.8 & 43.9 & 778 & 1,030 & 5.7 \\
\hline 84. & $\begin{array}{l}\mathrm{SE} 1 / 4 \mathrm{sec} .21, \mathrm{~T} .5 \mathrm{~S} ., \mathrm{R} .4 \mathrm{E} . \\
\text { River Raisin at Red Millpond, }\end{array}$ & 4.99 & 0 & 5.1 & 778 & 865 & 17.1 \\
\hline 85. & $\begin{array}{l}\text { SW1/4 sec. } 27, \text { T. } 5 \text { S., R. } 4 \text { E. } \\
\text { Evans Creek at Evans Lake }\end{array}$ & 222 & 88.7 & 45.0 & 770 & 1,030 & 5.7 \\
\hline 86. & $\begin{array}{l}\text { NE1/4 sec. 7, T. } 5 \text { S., R. } 3 \text { E. } \\
\text { Evans Creek at Tipton Road, }\end{array}$ & 4.28 & 14.2 & 3.1 & 959 & 968 & 2.9 \\
\hline 87. & $\begin{array}{l}\mathrm{NE}^{1 / 4} \text { sec. } 16, \mathrm{~T} .5 \mathrm{~S} ., \text { R. } 3 \mathrm{E} . \\
\text { Evans Creek at Wisner Road, }\end{array}$ & 12.1 & 9.8 & 7.5 & 893 & 968 & 10.0 \\
\hline 88. & $\begin{array}{l}\mathrm{NE}^{1 / 4} \text { sec. } 22, \mathrm{~T} .5 \mathrm{~S} ., \text { R. } 3 \mathrm{E} . \\
\text { Evans Creek above Bruno Drain, }\end{array}$ & 15.0 & 7.9 & 9.4 & 849 & 968 & 12.7 \\
\hline 89. & $\begin{array}{l}\text { SW } 1 / 4 \text { sec. } 23 \text {, T. } 5 \text { S., R. } 3 \text { E. } \\
\text { Bruno Drain at mouth, }\end{array}$ & 15.3 & 7.4 & 9.9 & 843 & 968 & 12.6 \\
\hline 90. & $\begin{array}{l}\mathrm{SW} 1 / 4 \mathrm{sec} .23, \mathrm{~T} .5 \mathrm{~S} ., \mathrm{R} .3 \mathrm{E} . \\
\text { Evans Creek at Bruno Drain, }\end{array}$ & 2.98 & 0 & 1.6 & 843 & 882 & 24.4 \\
\hline 91. & $\begin{array}{l}\text { SW1/4 sec. 23, T. } 5 \text { S., R. } 3 \text { E. } \\
\text { Evans Creek above Taylor Creek, }\end{array}$ & 18.3 & 7.4 & 9.9 & 843 & 968 & 12.6 \\
\hline 92. & $\begin{array}{l}\mathrm{SW} 1 / 4 \mathrm{sec} .24 \text {, T. } 5 \text { S., R. } 3 \text { E. } \\
\text { Tay lor Creek at mouth, }\end{array}$ & 18.7 & 6.5 & 10.8 & 835 & 968 & 12.3 \\
\hline & SW1/4 sec. 24, T. 5 S., R. 3 E. & 4.24 & 0 & 3.9 & 835 & 938 & 26.4 \\
\hline
\end{tabular}


PHYSIOGRAPHIC DATA FOR SELECTED SUBBASINS IN THE RIVER RAISIN BASIN - Continl`d

\begin{tabular}{|c|c|c|c|c|c|c|c|}
\hline \multirow{2}{*}{$\begin{array}{c}\text { Num- } \\
\text { ber } \\
\text { key }\end{array}$} & Stream and location & \multirow{2}{*}{$\begin{array}{l}\text { Drainage } \\
\text { area } \\
\text { (sq } \mathrm{mi})\end{array}$} & \multicolumn{2}{|c|}{ Miles } & \multicolumn{2}{|c|}{$\begin{array}{c}\text { Altitude } \\
\text { (ft above msl) }\end{array}$} & \multirow{2}{*}{$\begin{array}{l}\text { Average } \\
\text { slope } \\
\text { (ft per } \\
\text { mile) }\end{array}$} \\
\hline & 123456 Ranking order & & $\begin{array}{l}\text { above } \\
\text { mouth }\end{array}$ & $\begin{array}{l}\text { below } \\
\text { source }\end{array}$ & $\begin{array}{c}\text { at } \\
\text { site }\end{array}$ & $\begin{array}{c}\text { at } \\
\text { source }\end{array}$ & \\
\hline $\begin{array}{l}93 . \\
94 .\end{array}$ & $\begin{array}{l}\text { Evans Creek at Taylor Creek, } \\
\text { SW } 1 / 4 \text { sec. } 24 \text {, T. } 5 \text { S., R. } 3 \text { E. } \\
\text { Evans Creek above Lamkin Drain, }\end{array}$ & 22.9 & 6.5 & 10.8 & 835 & 968 & 12.3 \\
\hline 95 . & $\begin{array}{l}\mathrm{SW} 1 / 4 \text { sec. } 24, \text { T. } 5 \mathrm{~S} ., \text { R. } 3 \mathrm{E} . \\
\text { Lamkin Drain at mouth, }\end{array}$ & 23.0 & 6.4 & 10.9 & 834 & 968 & 12.3 \\
\hline 96. & $\begin{array}{l}\text { SW1 } 14 \text { sec. } 24, \text { T. } 5 \text { S., R. } 3 \text { E. } \\
\text { Evans Creek at Lamkin Drain, }\end{array}$ & 3.52 & 0 & 1.6 & 834 & 858 & 15.0 \\
\hline 97. & $\begin{array}{l}\text { SW1 } 1 / 4 \text { sec. } 24, \text { T. } 5 \text { S., R. } 3 \text { E. } \\
\text { Evans Creek at mouth, }\end{array}$ & 26.5 & 6.4 & 10.9 & 834 & 968 & 12.3 \\
\hline 98. & $\begin{array}{l}\mathrm{SW} 1 / 4 \mathrm{sec} .27, \text { T. } 5 \text { S., R. } 4 \text { E. } \\
\text { River Raisin at dam, }\end{array}$ & 34.2 & 0 & 17.3 & 760 & 968 & 12.0 \\
\hline 99. & $\begin{array}{l}\mathrm{NE}^{1 / 4} \mathrm{sec} .34, \mathrm{~T} .5 \mathrm{~S} ., \text { R. } 4 \mathrm{E} . \\
\text { River Raisin at Highway } 50 \text {, }\end{array}$ & 257 & 87.2 & 46.5 & 760 & 1,030 & 5.8 \\
\hline 100. & $\begin{array}{l}\text { NE } 1 / 4 \mathrm{sec} .34, \mathrm{~T} .5 \mathrm{~S} ., \mathrm{R} .4 \mathrm{E} \text {. } \\
\text { River Raisin at North Raisin Center Highway } \\
\text { (gage), }\end{array}$ & 259 & 87.1 & 46.6 & 745 & 1,030 & 6.1 \\
\hline 101. & $\begin{array}{l}\mathrm{NE} 1 / 4 \mathrm{sec} .21, \mathrm{~T} .6 \mathrm{~S} ., \text { R. } 4 \mathrm{E} \text {. } \\
\text { River Raisin above South Branch River Raisin, }\end{array}$ & 267 & 80.9 & 52.8 & 711 & 1,030 & 6.0 \\
\hline 102. & $\begin{array}{l}\mathrm{SW} 1 / 4 \text { sec. } 29, \text { T. } 6 \text { S., R. } 4 \text { E. } \\
\text { South Branch River Raisin above unnamed } \\
\text { tributary, }\end{array}$ & 270 & 77.5 & 56.2 & 699 & 1,030 & 5.9 \\
\hline 103. & $\begin{array}{l}\mathrm{SE}^{1} / 4 \text { sec. } 7, \mathrm{~T} .7 \mathrm{~S} ., \text { R. } 2 \mathrm{E} . \\
\text { Unnamed tributary at mouth, }\end{array}$ & 3.32 & 29.6 & 4.1 & 867 & 935 & 16.6 \\
\hline & $\begin{array}{l}\text { SE } 1 / 4 \text { sec. } 7, \text { T. } 7 \text { S., R. } 2 \text { E. } \\
\text { South Branch River Raisin at unnamed tributary, }\end{array}$ & 2.78 & 0 & 3.2 & 867 & 940 & 22.8 \\
\hline $\begin{array}{l}104 . \\
105 .\end{array}$ & $\begin{array}{l}\mathrm{SE} 1 / 4 \mathrm{sec} .7, \mathrm{~T} .7 \mathrm{~S} ., \mathrm{R} .2 \mathrm{E} \text {. } \\
\text { South Branch River Raisin above unnamed } \\
\text { tributary, }\end{array}$ & 6.10 & 29.6 & 4.1 & 867 & 935 & 16.6 \\
\hline \multirow{2}{*}{$\begin{array}{l}106 . \\
107 .\end{array}$} & $\begin{array}{l}\mathrm{SE}^{1 / 4} / 4 \mathrm{sec} .18, \mathrm{~T} .7 \mathrm{~S} ., \mathrm{R} .2 \mathrm{E} . \\
\text { Unnamed tributary at mouth, }\end{array}$ & 7.12 & 27.8 & 5.9 & 847 & 935 & 14.9 \\
\hline & $\begin{array}{l}\text { SE } 1 / 4 \text { sec. } 18, \text { T. } 7 \text { S., R. } 2 \text { E. } \\
\text { South Branch River Raisin, at unnamed tributary, }\end{array}$ & 1.66 & 0 & 2.8 & 847 & 910 & 22.5 \\
\hline 108 & $\begin{array}{l}\text { SE } 1 / 4 \text { sec. 18, T. } 7 \text { S., R. } 2 \text { E. } \\
\text { South Branch River Raisin above Bordine Drain, }\end{array}$ & 8.78 & 27.8 & 5.9 & 847 & 935 & 14.9 \\
\hline \multirow[t]{2}{*}{109.} & $\begin{array}{l}\text { NW1/4 sec. } 21, \text { T. } 7 \text { S., R. } 2 \text { E. } \\
\text { Bordine Drain at mouth, }\end{array}$ & 11.3 & 24.8 & 8.9 & 822 & 935 & 12.7 \\
\hline & $\begin{array}{l}\mathrm{NW} 1 / 4 \mathrm{sec} .21, \mathrm{~T} .7 \mathrm{~S} ., \text { R. } 2 \mathrm{E} \text {. } \\
\text { South Branch River Raisin at Bordine Drain, }\end{array}$ & 1.03 & 0 & 1.7 & 822 & $84 n$ & 10.6 \\
\hline $\begin{array}{l}110 . \\
111 .\end{array}$ & $\begin{array}{l}\text { NW11/4 sec. } 21, \text { T. } 7 \text { S., R. } 2 \text { E. } \\
\text { South Branch River Raisin above Harrison Drain, }\end{array}$ & 12.3 & 24.8 & 8.9 & 822 & 935 & 12.7 \\
\hline \multirow[t]{2}{*}{112.} & $\begin{array}{l}\text { NW1/4 sec. } 22, \mathrm{~T} .7 \mathrm{~S} ., \mathrm{R} .2 \mathrm{E} . \\
\text { Harrison Drain above Nash Drain, }\end{array}$ & 13.1 & 23.7 & 10.0 & 812 & 935 & 12.3 \\
\hline & $\begin{array}{l}\text { SW1/4 sec. } 5, \text { T. } 7 \text { S., R. } 2 \text { E. } \\
\text { Nash Drain at mouth, }\end{array}$ & 1.46 & 4.4 & 2.2 & 874 & 928 & 24.5 \\
\hline 113. & $\begin{array}{l}\mathrm{SW} 1 / 4 \text { sec. } 5, \mathrm{~T} .7 \mathrm{~S} ., \text { R. } 2 \mathrm{E} . \\
\text { Harrison Drain at Nash Drain, }\end{array}$ & 1.20 & 0 & 2.6 & 874 & 936 & 23.8 \\
\hline 114. & SW1/4 sec. 5, T. 7 S., R. 2 E. & 2.66 & 4.4 & 2.2 & 874 & 928 & 24.5 \\
\hline 115. & $\begin{array}{l}\text { Harrison Drain above unnamed tributary, } \\
\mathrm{SE}^{1} / 4 \mathrm{sec} .9, \mathrm{~T} .7 \mathrm{~S} ., \mathrm{R} .2 \mathrm{E} \text {. } \\
\text { Unnamed tributary at mouth, }\end{array}$ & 5.62 & 1.6 & 5.0 & 828 & 928 & 20.0 \\
\hline \multirow{2}{*}{$\begin{array}{l}117 . \\
118 .\end{array}$} & $\begin{array}{l}\mathrm{SE}^{1 / 4} \mathrm{sec} .9, \mathrm{~T} .7 \mathrm{~S} ., \mathrm{R} .2 \mathrm{E} . \\
\text { Harrison Drain at unnamed tributary, }\end{array}$ & 1.64 & 0 & 1.6 & 828 & 864 & 22.5 \\
\hline & $\begin{array}{l}\mathrm{SE}^{1 / 4} \mathrm{sec} .9, \mathrm{~T} .7 \mathrm{~S} ., \mathrm{R} .2 \mathrm{E} \text {. } \\
\text { Harrison Drain at mouth, }\end{array}$ & 7.26 & 1.6 & 5.0 & 828 & 928 & 20.0 \\
\hline \multirow{3}{*}{$\begin{array}{l}119 . \\
120 .\end{array}$} & $\begin{array}{l}\text { NW1/4 sec. } 22 \text {, T. } 7 \text { S., R. } 2 \text { E. } \\
\text { South Branch River Raisin at Harrison Drain, }\end{array}$ & 8.46 & 0 & 6.6 & 812 & 928 & 17.6 \\
\hline & $\begin{array}{l}\text { NW1/4 sec. 22, T. } 7 \text { S., R. } 2 \text { E. } \\
\text { South Branch River Raisin at Sword Highway, }\end{array}$ & 21.5 & 23.7 & 10.0 & 812 & 935 & 12.3 \\
\hline & NE $1 / 4$ sec. 14, T. 7 S., R. 2 E. & 23.7 & 21.5 & 12.2 & 795 & 935 & 11.5 \\
\hline
\end{tabular}


PHYSIOGRAPHIC DATA FOR SELECTED SUBBASINS IN THE RIVER RAISIN BASIN - Continued

\begin{tabular}{c|c|}
\hline \multirow{2}{*}{$\begin{array}{c}\text { Num- } \\
\text { ber } \\
\text { key }\end{array}$} & $123456 \quad$ Ranking order \\
\cline { 2 - 3 } &
\end{tabular}

\begin{tabular}{|c|c|c|c|c|c|}
\hline \multirow{2}{*}{$\begin{array}{c}\text { Drainage } \\
\text { area } \\
\text { (sq } \mathrm{mi})\end{array}$} & \multicolumn{2}{|c|}{ Miles } & \multicolumn{2}{|c|}{$\begin{array}{c}\text { Altitude } \\
\text { (ft above msl) }\end{array}$} & \multirow{2}{*}{$\begin{array}{c}\text { Average } \\
\text { slope } \\
\text { (ft per } \\
\text { mile) }\end{array}$} \\
\hline & $\begin{array}{l}\text { above } \\
\text { mouth }\end{array}$ & $\begin{array}{l}\text { below } \\
\text { source }\end{array}$ & $\begin{array}{l}\text { at } \\
\text { site }\end{array}$ & $\begin{array}{c}\text { at } \\
\text { source }\end{array}$ & \\
\hline
\end{tabular}

121. South Branch River Raisin above Cadmus Drain, NW $1 / 4$ sec. 13, T. 7 S., R. 2 E.

122. Cadmus Drain at mouth, NW $1 \frac{1}{4}$ sec. 13, T. 7 S., R. 2 E.

123. South Branch River Raisin at Cadmus Drain, NW $1 / 4$ sec. 13, T. 7 S., R. 2 E.

124. South Branch River Raisin above Hazen Creek, NW $1 / 4$ sec. 13, T. 7 S., R. 2 E.

125. Hazen Creek at dam, NE $1 / 4$ sec. 25, T. 6 S., R. 1 E.

126. Hazen Creek at North Hawkins Highway, NE $1 / 4$ sec. 30 , T. 6 S., R. 2 E.

127. Hazen Creek at Forrister Road, SW $1 / 4$ sec. 28 , T. 6 S., R. 2 E.

128. Hazen Creek above Wallace Drain, SW $1 \frac{1}{4}$ sec. 35 , T. 6 S., R. 2 E.

129. Wallace Drain above unnamed tributary, NW $1 / 4$ sec. 22 , T. 6 S., R. 2 E.

130. Unnamed tributary at mouth, NW $1 \frac{1}{4}$ sec. 22 , T. 6 S., R. 2 E.

131. Wallace Drain at unnamed tributary, NW $1 / 4$ sec. 22 , T. 6 S., R. 2 E.

132. Wallace Drain above Stoddard Drain, NW $1 \frac{1}{4}$ sec. 22 , T. 6 S., R. 2 E.

133. Stoddard Drain above unnamed tributary, NW $1 / 4$ sec. 15 , T. 6 S., R. 2 E.

134. Unnamed tributary at mouth, NW $1 / 4$ sec. 15 , T. 6 S., R. 2 E.

135. Stoddard Drain at unnamed tributary, NW $1 \frac{1}{4}$ sec. 15 , T. 6 S., R. 2 E.

136.

137.

138.

Stoddard Drain at mouth, NW $1 / 4$ sec. 22 , T. 6 S., R. 2 E.

Wallace Drain at Stoddard Drain, NW $1 / 4$ sec. 22 , T. 6 S., R. 2 E.

Wallace Drain at Hoddinott Road, NE $1 / 4$ sec. 27 , T. 6 S., R. 2 E.

139. Penrod Drain at mouth,

140. SE1/4 sec. 27, T. 6 S., R. 2 E.

Wallace Drain at Penrod Drain, SE $1 / 4$ sec. 27 , T. 6 S., R. 2 E.

141. Wallace Drain at mouth, SW1 14 sec. 35, T. 6 S., R. 2 E.

142. Hazen Creek at Wallace Drain, SW $1 / 4$ sec. 35, T. 6 S., R. 2 E.

143. Hazen Creek above Lowery Drain, NW $1 \frac{1}{4}$ sec. 11, T. 7 S., R. 2 E.

144. Lowery Drain at mouth, NW $1 / 4$ sec. 11, T. 7 S., R. 2 E.

145. Hazen Creek at Lowery Drain, NW1/4 sec. 11, T. 7 S., R. 2 E.

146. Hazen Creek at Cadmus Road, SW $1 / 4$ sec. 12 , T. 7 S., R. 2 E.

147. Hazen Creek at mouth, NW1/4 sec. 13, T. 7 S., R. 2 E.

148. South Branch River Raisin at Hazen Creek, NW1/4 sec. 13, T. 7 S., R. 2 E.

149. South Branch River Raisin above Warren Drain, NE $1 / 4$ sec. 25 , T. 7 S., R. 2 E.

150. Warren Drain at mouth, NE $1 / 4$ sec. 25, T. 7 S., R. 2 E.

\begin{tabular}{|c|c|c|c|c|c|}
\hline 23.9 & 21.2 & 12.5 & 793 & 935 & 11.4 \\
\hline 1.42 & 0 & 1.4 & 793 & 825 & 22.9 \\
\hline 25.3 & 21.2 & 12.5 & 793 & 935 & 11.4 \\
\hline 25.4 & 21.0 & 12.7 & 792 & 935 & 11.3 \\
\hline 3.60 & 10.6 & 3.9 & 960 & 1,064 & 26.7 \\
\hline 5.07 & 9.0 & 5.5 & 921 & 1,064 & 26.0 \\
\hline 6.64 & 7.0 & 7.5 & 890 & 1,064 & 23.2 \\
\hline 8.95 & 4.1 & 10.4 & 836 & 1,064 & 21.9 \\
\hline 3.64 & 4.4 & 3.5 & 891 & 1,040 & 42.6 \\
\hline 1.33 & 0 & 2.0 & 891 & 985 & 47.0 \\
\hline 4.97 & 4.4 & 3.5 & 891 & 1,040 & 42.6 \\
\hline 5.03 & 4.2 & 3.7 & 888 & 1,040 & 41.1 \\
\hline 1.09 & .7 & 1.9 & 898 & 1,010 & 58.9 \\
\hline 1.02 & 0 & 1.8 & 898 & 979 & 45.0 \\
\hline 2.11 & .7 & 1.9 & 898 & 1,010 & 58.9 \\
\hline 2.91 & 0 & 2.6 & 888 & 1,010 & 46.9 \\
\hline 7.94 & 4.2 & 3.7 & 888 & 1,040 & 41.1 \\
\hline 10.1 & 1.9 & 6.0 & 858 & 1,040 & 30.3 \\
\hline .74 & 0 & 1.4 & 858 & 915 & 40.7 \\
\hline 10.9 & 1.9 & 6.0 & 858 & 1,040 & 30.3 \\
\hline 11.7 & 0 & 7.9 & 836 & 1,040 & 25.8 \\
\hline 20.7 & 4.1 & 10.4 & 836 & 1,064 & 21.9 \\
\hline 22.1 & 2.2 & 12.3 & 810 & 1,064 & 20.7 \\
\hline .66 & 0 & 1.4 & 810 & 853 & 30.7 \\
\hline 22.8 & 2.2 & 12.3 & 810 & 1,064 & 20.7 \\
\hline 24.9 & .3 & 14.2 & 794 & 1,064 & 19.0 \\
\hline 25.0 & 0 & 14.5 & 792 & 1,064 & 18.8 \\
\hline 50.4 & 21.0 & 12.7 & 792 & 935 & 11.3 \\
\hline 53.4 & 17.4 & 16.3 & 775 & 935 & 9.8 \\
\hline 1.70 & 0 & .7 & 775 & 792 & 24.3 \\
\hline
\end{tabular}


PHYSIOGRAPHIC DATA FOR SELECTED SUBBASINS IN THE RIVER RAISIN BASIN - Continıed

\begin{tabular}{|c|c|}
\hline \multirow{2}{*}{$\begin{array}{l}\text { Num- } \\
\text { ber } \\
\text { key }\end{array}$} & Stream and location \\
\hline & 123456 Ranking order \\
\hline
\end{tabular}

\begin{tabular}{|c|c|c|c|c|c|}
\hline \multirow{2}{*}{$\begin{array}{c}\text { Drainage } \\
\text { area } \\
\text { (sq } \mathrm{mi} \text { ) }\end{array}$} & \multicolumn{2}{|c|}{ Miles } & \multicolumn{2}{|c|}{$\begin{array}{c}\text { Altitude } \\
\text { (ft above msl) }\end{array}$} & \multirow{2}{*}{$\begin{array}{c}\text { Average } \\
\text { slope } \\
\text { (ft per } \\
\text { mile) }\end{array}$} \\
\hline & $\begin{array}{l}\text { above } \\
\text { mouth }\end{array}$ & $\begin{array}{l}\text { below } \\
\text { source }\end{array}$ & $\begin{array}{l}\text { at } \\
\text { site }\end{array}$ & $\begin{array}{c}\text { at } \\
\text { source }\end{array}$ & \\
\hline
\end{tabular}

151. South Branch River Raisin at Warren Drain,

152. South Branch River Raisin above Stony Creek,
Sec. 25, T. 7 S., R. 2 E. NE $1 / 4$ sec. 31 , T. 7 S., R. 3 E.

153. Big Swamp Drain (headwaters of Stony Creek) above Bovee Drain, NE $1 / 4$ sec. 33 , T. 7 S., R. 2 E.

154. Bovee Drain at mouth, NE $1 / 4$ sec. 33, T. 7 S., R. 2 E.

155. Big Swamp Drain at Bovee Drain, NE $1 / 4$ sec. 33, T. 7 S., R. 2 E.

156. Stony Creek at Benner Highway, NE $1 / 4$ sec. 34, T. 7 S., R. 2 E.

157. Stony Creek above unnamed tributary, SW $1 \frac{1}{4}$ sec. 36, T. 7 S., R. 2 E.

158. Unnamed tributary at mouth, SW $1 / 4$ sec. 36, T. 7 S., R. 2 E.

159. Stony Creek at unnamed tributary, SW $1 / 4$ sec. 36, T. 7 S., R. 2 E.

160. Stony Creek above South Branch Stony Creek, SW $1 / 4$ sec. 31, T. 7 S., R. 3 E.

161. South Branch Stony Creek above Complex Drain, NE $1 / 4$ sec. 1, T. 8 S., R. 2 E.

162. Complex Drain at mouth, $\mathrm{NE}^{\prime 1 / 4}$ sec. 1 , T. 8 S., R. 2 E.

163. South Branch Stony Creek at Complex Drain, NE $1 / 4$ sec. 1, T. 8 N., R. 2 E.

164. South Branch Stony Creek at mouth, SW1/4 sec. 31, T. 7 S., R. 3 E.

165. Stony Creek at South Branch Stony Creek, SW $1 / 4$ sec. 31 , T. 7 S., R. 3 E.

166. Stony Creek at Livesay Road, NE $1 / 4$ sec. 31 , T. 7 S., R. 3 E.

167. Stony Creek at mouth, NE $1 / 4$ sec. 31 , T. 7 S., R. 3 E.

168. South Branch River Raisin at Stony Creek, NE $1 / 4$ sec. 31 , T. 7 S., R. 3 E.

169. South Branch River Raisin at Sand Creek Highway, NE $1 / 4$ sec. 29, T. 7 S., R. 3 E.

170. South Branch River Raisin above Porter Drain, NW1/4 sec. 22, T. 7 S., R. 3 E.

171. Porter Drain at West Carleton Road, SE $1 / 4$ sec. 17 , T. 7 S., R. 3 E.

172. Porter Drain at mouth, NW1/4 sec. 22 , T. 7 S., R. 3 E.

173. South Branch River Raisin at Porter Drain, NW $1 / 4$ sec. 22, T. 7 S., R. 3 E.

174. South Branch River Raisin above Savage Drain, NE $1 \frac{1}{4}$ sec. 15, T. 7 S., R. 3 E.

175. Savage Drain at mouth, NE $1 / 4$ sec. 15 , T. 7 S., R. 3 E.

176. South Branch River Raisin at Savage Drain, NE $1 / 4$ sec. 15, T. 7 S., R. 3 E.

177. South Branch River Raisin at Highway 223, SE $1 / 4$ sec. 10, T. 7 S., R. 3 E.

178. South Branch River Raisin at Beecher Street NE $1 / 4$ sec. 10, T. 7 S., R. 3 E.

\begin{tabular}{|c|c|c|c|c|c|}
\hline 55.1 & 17.4 & 16.3 & 775 & 935 & 9.8 \\
\hline 57.4 & 14.4 & 19.3 & 764 & 935 & 8.9 \\
\hline 2.14 & 5.1 & 2.2 & 801 & 811 & 4.5 \\
\hline 1.38 & 0 & 1.4 & 801 & 827 & 13.6 \\
\hline 3.52 & 5.1 & 2.2 & 801 & 811 & 4.5 \\
\hline 4.55 & 4.1 & 3.2 & 795 & 811 & 5.0 \\
\hline 6.59 & 1.8 & 5.5 & 775 & 811 & 6.5 \\
\hline 1.87 & 0 & 1.9 & 775 & 807 & 13.2 \\
\hline 8.46 & 1.8 & 5.5 & 775 & 811 & 6.5 \\
\hline 8.86 & 1.3 & 6.0 & 771 & 811 & 6.7 \\
\hline 1.54 & .5 & 1.4 & 773 & $78 n$ & 5.0 \\
\hline 1.23 & 0 & 2.0 & 773 & $78 ?$ & 8.0 \\
\hline 2.77 & .5 & 1.4 & 773 & 780 & 5.0 \\
\hline 3.13 & 0 & 1.9 & 771 & 780 & 4.7 \\
\hline 12.0 & 1.3 & 6.0 & 771 & 811 & 6.7 \\
\hline 12.9 & .1 & 7.2 & 765 & 811 & 6.4 \\
\hline 12.9 & 0 & 7.3 & 764 & 811 & 6.4 \\
\hline 70.3 & 14.4 & 19.3 & 764 & 935 & 8.9 \\
\hline 71.8 & 12.3 & 21.4 & 755 & 935 & 8.4 \\
\hline 75.2 & 9.1 & 24.6 & 745 & 935 & 7.7 \\
\hline 3.01 & 1.8 & 2.3 & 781 & $79^{\prime}$ & 7.8 \\
\hline 3.67 & 0 & 4.1 & 745 & $79^{n}$ & 10.7 \\
\hline 78.9 & 9.1 & 24.6 & 745 & 935 & 7.7 \\
\hline 80.2 & 7.9 & 25.8 & 742 & 935 & 7.5 \\
\hline 2.83 & 0 & 3.0 & 742 & 790 & 16.0 \\
\hline 83.1 & 7.9 & 25.8 & 742 & 935 & 7.5 \\
\hline 85.9 & 7.3 & 26.4 & 739 & 935 & 7.4 \\
\hline 86.8 & 6.3 & 27.4 & 736 & 935 & \\
\hline
\end{tabular}


PHYSIOGRAPHIC DATA FOR SELECTED SUBBASINS IN THE RIVER RAISIN BASIN -Continued

\begin{tabular}{l|l|}
\hline \multirow{2}{*}{$\begin{array}{r}\text { Num- } \\
\text { ber }\end{array}$} & \multicolumn{1}{|c|}{ Stream and location } \\
\cline { 2 - 2 } key & $123456 \quad$ Ranking order \\
\hline
\end{tabular}

\begin{tabular}{|c|c|c|c|c|c|}
\hline \multirow{2}{*}{$\begin{array}{l}\text { Drainage } \\
\text { area } \\
(\mathrm{sq} \mathrm{mi})\end{array}$} & \multicolumn{2}{|c|}{ Miles } & \multicolumn{2}{|c|}{$\begin{array}{c}\text { Altitude } \\
\text { (ft above } \mathrm{msl} \text { ) }\end{array}$} & \multirow{2}{*}{$\begin{array}{c}\text { Average } \\
\text { slope } \\
\text { (ft per } \\
\text { mile) }\end{array}$} \\
\hline & $\begin{array}{l}\text { above } \\
\text { mouth }\end{array}$ & $\begin{array}{l}\text { below } \\
\text { source }\end{array}$ & $\begin{array}{l}\text { at } \\
\text { site }\end{array}$ & $\begin{array}{c}\text { at } \\
\text { source }\end{array}$ & \\
\hline
\end{tabular}

179. South Branch River Raisin above Wolf Creek, NE $\frac{1}{4}$ sec. 35, T. 6 S., R. 3 E.

180. Wolf Creek at Springville Road, SE $1 / 4$ sec. 15, T. 5 S., R. 2 E.

181. Wolf Creek at Gilbert Highway, NW $1 / 4$ sec. 36, T. 5 S., R. 2 E.

182. Wolf Creek above Squaw Creek, NE $1 / 4$ sec. 35 , T. 5 S., R. 2 E.

183. Squaw Creek above unnamed tributary, SW $1 \frac{1}{4}$ sec. 32, T. 5 S., R. 2 E.

184. Unnamed tributary at mouth, SW $1 / 4$ sec. 32 , T. 5 S., R. 2 E.

185. Squaw Creek at unnamed tributary, SW1 14 sec. 32, T. 5 S., R. 2 E.

186. Squaw Creek above unnamed tributary, NW $1 / 4$ sec. 3, T. 6 S., R. 2 E.

187. Unnamed tributary at mouth, NW $1 \frac{1}{4}$ sec. 3, T. 6 S., R. 2 E.

188. Squaw Creek at unnamed tributary, NW $1 \frac{1}{4}$ sec. 3, T. 6 S., R. 2 E.

189. Squaw Creek at dam, NE $1 / 4$ sec. 3, T. 6 S., R. 2 E.

190. Squaw Creek above unnamed tributary, SW1 $1 / 4$ sec. 35 , T. 5 S., R. 2 E.

191. Unnamed tributary at mouth, SW $1 \frac{4}{4}$ sec. 35 , T. 5 S., R. 2 E.

192. Squaw Creek at unnamed tributary, SW1/4 sec. 35, T. 5 S., R. 2 E.

193. Squaw Creek at Teachout Road, NW $1 / 4$ sec. 2 , T. 6 S., R. 2 E.

194. Squaw Creek at mouth, NE $\frac{1}{4}$ sec. 35 , T. 5 S., R. 2 E.

195. Wolf Creek at Squaw Creek, NE $1 / 4$ sec. 35 , T. 5 S., R. 2 E.

196. Wolf Creek at Shepherd Road, SW $1 / 4$ sec. 6, T. 6 S., R. 3 E.

197. Wolf Creek above Fouty Creek, NW $1 \frac{1}{4}$ sec. 7, T. 6 S., R. 3 E.

198. Fouty Creek above Gilbert Drain, NE $1 / 4$ sec. 12, T. 6 S., R. 2 E.

199. Gilbert Drain at mouth, NE $1 / 4$ sec. 12, T. 6 S., R. 2 E.

200. Fouty Creek at Gilbert Drain, NE $1 / 4$ sec. 12, T. 6 S., R. 2 E.

201. Fouty Creek at mouth, NW $1 / 4$ sec. 7, T. 6 S., R. 3 E.

202. Wolf Creek at Fouty Creek, NW $1 / 4$ sec. 7 , T. 6 S., R. 3 E.

203. Wolf Creek above Ryan Creek, NW $1 / 4$ sec. 18 , T. 6 S., R. 3 E.

204. Ryan Creek at mouth, NW1/4 sec. 18 , T. 6 S., R. 3 E.

205. Wolf Creek at Ryan Creek, NW $1 / 4$ sec. 18, T. 6 S., R. 3 E.

206. Wolf Creek above Hunt Creek, SW $1 / 4$ sec. 19, T. 6 S., R. 3 E.

207. Hunt Creek above Atwood Drain, $\mathrm{SE} 1 / 4$ sec. 24, T. 6 S., R. 2 E.

208. Atwood Drain at mouth, SE $1 / 4$ sec. 24 , T. 6 S., R. 2 E.

\begin{tabular}{|c|c|c|c|c|}
\hline 89.7 & 4.1 & 29.6 & 726 & 935 \\
\hline 6.39 & 25.9 & 5.1 & 931 & 994 \\
\hline 18.1 & 22.3 & 8.7 & 900 & 994 \\
\hline 18.7 & 21.8 & 9.2 & 894 & 994 \\
\hline 3.37 & 5.3 & 2.1 & 1,008 & 1,068 \\
\hline 1.02 & 0 & 2.2 & 1,008 & 1,047 \\
\hline 4.39 & 5.3 & 2.1 & 1,008 & 1,068 \\
\hline 7.86 & 2.2 & 5.2 & 939 & 1,068 \\
\hline .79 & 0 & 1.9 & 939 & 1,005 \\
\hline 8.65 & 2.2 & 5.2 & 939 & 1,068 \\
\hline 8.98 & 1.6 & 5.8 & 931 & 1,068 \\
\hline 9.16 & 1.2 & 6.2 & 913 & 1,068 \\
\hline 1.54 & 0 & 3.2 & 913 & 997 \\
\hline 10.7 & 1.2 & 6.2 & 913 & 1,068 \\
\hline 11.0 & .7 & 6.7 & 903 & 1,068 \\
\hline 11.4 & 0 & 7.4 & 894 & 1,068 \\
\hline 30.2 & 21.8 & 9.2 & 894 & 994 \\
\hline 32.6 & 18.2 & 12.8 & 872 & 994 \\
\hline 32.7 & 17.8 & 13.2 & 868 & 994 \\
\hline .58 & .4 & 1.4 & 878 & 926 \\
\hline 1.38 & 0 & 2.0 & 878 & 939 \\
\hline 1.96 & .4 & 1.4 & 878 & 926 \\
\hline 2.12 & 0 & 1.8 & 868 & 926 \\
\hline 34.8 & 17.8 & 13.2 & 868 & 994 \\
\hline 36.9 & 14.5 & 16.5 & 846 & 994 \\
\hline 1.27 & 0 & 1.9 & 846 & 900 \\
\hline 38.2 & 14.5 & 16.5 & 846 & 994 \\
\hline 39.4 & 12.1 & 18.9 & 833 & 994 \\
\hline .94 & .8 & 1.4 & 854 & 899 \\
\hline .61 & 0 & 1.1 & 854 & 895 \\
\hline
\end{tabular}


PHYSIOGRAPHIC DATA FOR SELECTED SUBBASINS IN THE RIVER RAISIN BASIN - Continued

\begin{tabular}{c|c|}
\hline \multirow{2}{*}{$\begin{array}{c}\text { Num- } \\
\text { ber } \\
\text { key }\end{array}$} & $123456 \quad$ Ranking order \\
\cline { 2 - 3 } &
\end{tabular}

\begin{tabular}{c|c} 
Drainage \\
area \\
(sq $\mathrm{mi})$
\end{tabular}

\begin{tabular}{|c|c|c|c|}
\hline \multicolumn{2}{|c|}{ Miles } & \multicolumn{2}{c|}{$\begin{array}{c}\text { Altitude } \\
\text { (ft above msl) }\end{array}$} \\
\hline $\begin{array}{c}\text { above } \\
\text { mouth }\end{array}$ & $\begin{array}{l}\text { below } \\
\text { source }\end{array}$ & $\begin{array}{c}\text { at } \\
\text { site }\end{array}$ & $\begin{array}{c}\text { at } \\
\text { source }\end{array}$ \\
\hline
\end{tabular}

$209 . \quad$ Hunt Creek at Atwood Drain, SE $1 / 4$ sec. 24, T. 6 S., R. 2 E.

210. Hunt Creek at mouth, SW1 $\frac{1}{4}$ sec. 19, T. 6 S., R. 3 E.

211. Wolf Creek at Hunt Creek, SW $1 \frac{1}{4}$ sec. 19, T. 6 S., R. 3 E.

212. Wolf Creek at Burton Road, SW $1 / 4$ sec. 19 , T. 6 S., R. 3 E.

213. Wolf Creek above Torry Drain, NW $1 / 4$ sec. 30, T. 6 S., R. 3 E.

214. Torry Drain at mouth, NW $1 / 4$ sec. 30 , T. 6 S., R. 3 E.

215. Wolf Creek at Torry Drain, NW $1 / 4$ sec. 30, T. 6 S., R. 3 E.

216. Wolf Creek above Black Creek NW $1 / 4$ sec. 30, T. 6 S., R. 3 E.

217. Black Creek at Shepherd Road, $\mathrm{SE}^{1 / 4} \mathrm{sec}$. 4, T. $6 \mathrm{~S}$., R. $3 \mathrm{E}$.

218. Black Creek above Fisk Drain, SW $1 / 4$ sec. 9, T. 6 S., R. 3 E.

219. Fisk Drain at mouth, SW $1 / 4$ sec. 9, T. 6 S., R. 3 E.

220. Black Creek at Fisk Drain, SW $1 / 4$ sec. 9, T. 6 S., R. 3 E.

221. Black Creek above unnamed tributary, SW $1 / 4$ sec. 17, T. 6 S., R. 3 E.

222.

Unnamed tributary at mouth, SW $1 / 4$ sec. 17 , T. 6 S., R. 3 E.

223. Black Creek at unnamed tributary, SW $1 / 4$ sec. 17 , T. 6 S., R. 3 E.

224. Black Creek at Burton Road, SW $1 / 4$ sec. 19, T. 6 S., R. 3 E.

225. Black Creek at mouth, NW $1 / 4$ sec. 30, T. 6 S., R. 3 E.

226.

Wolf Creek at Black Creek, NW $1 / 4$ sec. 30 , T. 6 S., R. 3 E.

227. Wolf Creek above Hood Drain NW1/4 sec. 31 , T. 6 S., R. 3 E.

228. Pickford Drain (headwaters of Hood Drain)

\begin{tabular}{|c|c|c|c|c|c|}
\hline 1.55 & 0.8 & 1.4 & 854 & 899 & 32.1 \\
\hline 1.94 & 0 & 2.2 & 833 & 899 & 30.0 \\
\hline 41.4 & 12.1 & 18.9 & 833 & 994 & 8.5 \\
\hline 41.4 & 11.7 & 19.3 & 831 & 994 & 8.4 \\
\hline 41.6 & 11.3 & 19.7 & 827 & 994 & 8.5 \\
\hline .44 & 0 & .9 & 827 & 859 & 35.6 \\
\hline 42.0 & 11.3 & 19.7 & 827 & 994 & 8.5 \\
\hline 42.0 & 11.2 & 19.8 & 827 & 994 & 8.4 \\
\hline 8.94 & 5.0 & 4.1 & 862 & 949 & 21.2 \\
\hline 9.96 & 3.9 & 5.2 & 854 & 949 & 18.3 \\
\hline 3.20 & 0 & 2.6 & 854 & 880 & 10.0 \\
\hline 13.2 & 3.9 & 5.2 & 854 & 949 & 18.3 \\
\hline 14.7 & 2.3 & 6.8 & 846 & 949 & 15.1 \\
\hline .83 & 0 & 1.3 & 846 & 867 & 16.2 \\
\hline 15.5 & 2.3 & 6.8 & 846 & 949 & 15.1 \\
\hline 16.7 & .2 & 8.9 & 829 & 949 & 13.5 \\
\hline 16.7 & 0 & 9.1 & 827 & 949 & \\
\hline 58.7 & 11.2 & 19.8 & 827 & 994 & \\
\hline 60.0 & 8.2 & 22.8 & 810 & 994 & \\
\hline
\end{tabular}

at Highway 223, SW $1 \frac{1}{4}$ sec. 25, T. 6 S., R. 2 E.

229. Hood Drain at mouth, NW $1 \frac{4}{4}$ sec. 31 , T. 6 S., R. 3 E.

230. Wolf Creek at Hood Drain, NW $1 / 4$ sec. 31 , T. 6 S., R. 3 E

231. Wolf Creek above unnamed tributary, SW $1 / 4$ sec. 28 , T. 6 S., R. 3 E.

232. Unnamed tributary at mouth, SW $1 / 4$ sec. 28, T. 6 S., R. 3 E.

233. Wolf Creek at unnamed tributary, SW $1 \frac{1}{4}$ sec. 28 , T. 6 S., R. 3 E.

234. Wolf Creek above Turner Drain, SW $1 / 4$ sec. 27 , T. 6 S., R. 3 E.

235. Turner Drain above unnamed tributary, NE $1 / 4$ sec. 28 , T. 6 S., R. 3 E.

236.

Unnamed tributary at mouth, NE $1 / 4$ sec. 28 , T. 6 S., R. 3 E.

237. Turner Drain at unnamed tributary, NE $1 / 4$ sec. 28 , T. 6 S., R. 3 E.

\begin{tabular}{clrrrr}
1.80 & 1.4 & 1.8 & 843 & 892 & 27.2 \\
3.02 & \multicolumn{1}{l}{0} & 3.2 & 810 & 892 & 23.1 \\
63.0 & .8 .2 & 22.8 & 810 & 994 & 7.7 \\
66.5 & \multicolumn{1}{c}{4.6} & 26.4 & 776 & 994 & 8.3 \\
.87 & \multicolumn{1}{c}{0} & 1.3 & 776 & 836 & 46.2 \\
67.3 & \multicolumn{1}{c}{4.6} & 26.4 & 776 & 994 & 8.3 \\
69.7 & \multicolumn{1}{c}{3.0} & 28.0 & 763 & 994 & 8.3 \\
1.83 & .5 & 1.7 & 780 & 827 & 27.6 \\
.88 & \multicolumn{1}{c}{0} & 1.0 & 780 & 825 & 45.0 \\
2.71 & .5 & 1.7 & 780 & 827 & 27.6
\end{tabular}


PHYSIOGRAPHIC DATA FOR SELECTED SUBBASINS IN THE RIVER RAISIN BASIN - Continued

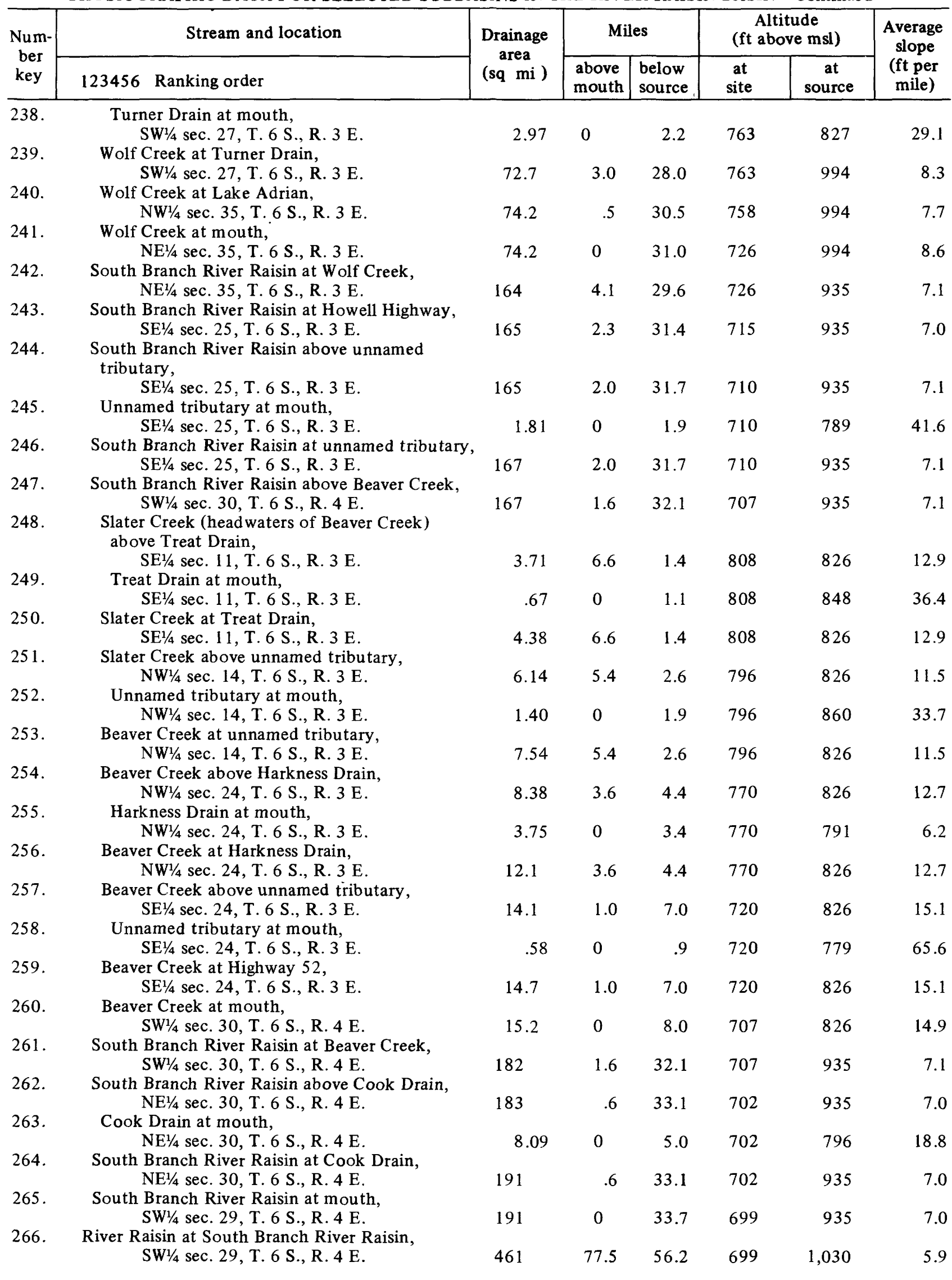


PHYSIOGRAPHIC DATA FOR SELECTED SUBBASINS IN THE RIVER RAISIN BASIN - Continued

\begin{tabular}{|c|c|c|c|c|c|c|c|}
\hline \multirow{2}{*}{$\begin{array}{c}\text { Num- } \\
\text { ber } \\
\text { key }\end{array}$} & Stream and location & \multirow{2}{*}{$\begin{array}{l}\text { Drainage } \\
\text { area } \\
\text { (sq } \mathrm{mi} \text { ) }\end{array}$} & \multicolumn{2}{|c|}{ Miles } & \multicolumn{2}{|c|}{$\begin{array}{c}\text { Altitude } \\
\text { (ft above msl) }\end{array}$} & \multirow{2}{*}{$\begin{array}{c}\text { Average } \\
\text { slope } \\
\text { (ft per } \\
\text { mile) }\end{array}$} \\
\hline & 123456 Ranking order & & $\begin{array}{l}\text { above } \\
\text { mouth }\end{array}$ & $\begin{array}{l}\text { below } \\
\text { source }\end{array}$ & $\begin{array}{l}\text { at } \\
\text { site }\end{array}$ & $\begin{array}{c}\text { at } \\
\text { source }\end{array}$ & \\
\hline 267. & $\begin{array}{l}\text { River Raisin at Academy Road (gage), } \\
\text { NW1/4 sec. } 5, \text { T. } 7 \text { S., R. } 4 \text { E. }\end{array}$ & 463 & 75.0 & 58.7 & 697 & 1,030 & 5.7 \\
\hline 268. & $\begin{array}{l}\text { River Raisin at Palmyra, } \\
\text { NW1/4 sec. 22, T. } 7 \text { S., R. } 4 \text { E. } \\
\text { River Raisin above Black Creek. }\end{array}$ & 467 & 66.9 & 66.8 & 683 & 1,030 & 5.2 \\
\hline $\begin{array}{l}269 . \\
270 .\end{array}$ & $\begin{array}{l}\text { NW1 } 1 / 4 \text { sec. } 2 \text {, T. } 8 \text { S., R. } 4 \text { E. } \\
\text { Bear Creek (headwaters of Black Creek), at } \\
\text { West Cadmus Road, }\end{array}$ & 471 & 60.5 & 73.2 & 677 & 1,030 & 4.8 \\
\hline 271. & $\begin{array}{l}\text { SW } 1 / 4 \text { sec. } 10 \text {, T. } 7 \text { S., R. } 1 \text { E. } \\
\text { Bear Creek above Henning Drain, }\end{array}$ & 2.83 & 36.8 & 2.6 & 914 & 969 & 21.2 \\
\hline 272. & $\begin{array}{l}\text { NW } 1 / 4 \text { sec. } 14, T .7 \text { S., R. } 1 \text { E. } \\
\text { Henning Drain at mouth, }\end{array}$ & 3.15 & 35.8 & 3.6 & 894 & 969 & 20.8 \\
\hline 73. & $\begin{array}{l}\text { NW1/4 Sec. } 14, \mathrm{~T} .7 \mathrm{~S} ., \text { R. } 1 \text { E. } \\
\text { Bear Creek at Henning Drain, }\end{array}$ & .57 & 0 & 1.1 & 894 & 929 & 31.8 \\
\hline 274. & $\begin{array}{l}\mathrm{NW}^{1 / 4} \mathrm{sec} .14, \mathrm{~T} .7 \mathrm{~S} ., \text { R. } 1 \mathrm{E} . \\
\text { Bear Creek at Highway } 34 \text {, }\end{array}$ & 3.72 & 35.8 & 3.6 & 894 & 969 & 20.8 \\
\hline 275. & $\begin{array}{l}\mathrm{SE}^{1 / 4} \mathrm{sec} .14, \mathrm{~T} .7 \mathrm{~S} ., \mathrm{R} .1 \mathrm{E} . \\
\text { Camp Drain at mouth, }\end{array}$ & 4.56 & 34.8 & 4.6 & 873 & 969 & 20.9 \\
\hline 76. & $\begin{array}{l}\text { SE1/4 sec. 26, T. } 7 \text { S., R. } 1 \text { E. } \\
\text { Bear Creek at Covell Lake, }\end{array}$ & 1.44 & 0 & 1.6 & 828 & 877 & 30.6 \\
\hline 77. & $\begin{array}{l}\text { SE } 1 / 4 \text { sec. } 26, \text { T. } 7 \text { S., R. } 1 \text { E. } \\
\text { J B Drain at mouth, }\end{array}$ & 8.53 & 31.5 & 7.9 & 828 & 969 & 17.8 \\
\hline & SE $1 / 4$ sec. 26 , T. 7 S., R. 1 E. & 1.42 & 0 & 2.4 & 828 & 866 & 15.8 \\
\hline 278. & 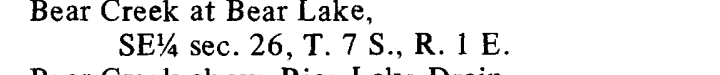 & 10.2 & 31.0 & 8.4 & 828 & 969 & 16.8 \\
\hline $\begin{array}{l}279 . \\
280 .\end{array}$ & $\begin{array}{l}\text { Bear Creek above Rice Lake Drain, } \\
\text { SW1/4 sec. } 31 \text {, T. } 7 \text { S., R. } 2 \text { E. } \\
\text { Rice Lake Drain at mouth, }\end{array}$ & 11.6 & 28.9 & 10.5 & 818 & 969 & 14.4 \\
\hline 281. & $\begin{array}{l}\text { SW1/4 sec. } 31, \mathrm{~T} .7 \text { S., R. } 2 \text { E. } \\
\text { Bear Creek at Rice Lake Drain, }\end{array}$ & 1.75 & 0 & 2.3 & 818 & 834 & 7.0 \\
\hline 282. & & 13.4 & 28.9 & 10.5 & 818 & 969 & 14.4 \\
\hline 283. & $\begin{array}{l}\text { SW1/4 sec. } 31, \text { T. } 7 \text { S., R. } 2 \text { E. } \\
\text { Bear Creek at Seneca Road, }\end{array}$ & 13.5 & 28.8 & 10.6 & 814 & 969 & 14.6 \\
\hline 284. & $\begin{array}{l}\text { SE } 1 / 4 \text { sec. } 4, \text { T. } 8 \text { S., R. } 2 \text { E. } \\
\text { Bear Creek at Packard Road, }\end{array}$ & 17.1 & 24.8 & 14.6 & 781 & 969 & 12.9 \\
\hline 285. & $\begin{array}{l}\text { SW1 } 1 / 4 \text { sec. } 11, \text { T. } 8 \text { S., R. } 2 \text { E. } \\
\text { Bear Creek at Elliott Road, }\end{array}$ & 20.2 & 22.3 & 17.1 & 769 & 969 & 11.7 \\
\hline 286. & $\begin{array}{l}\mathrm{SE}^{1 / 4} \text { sec. } 14, \mathrm{~T} .8 \mathrm{~S} ., \mathrm{R} .2 \mathrm{E} \\
\text { Black Creek above unnamed tributary, }\end{array}$ & 24.5 & 20.4 & 19.0 & 758 & 969 & 11.1 \\
\hline 287. & $\begin{array}{l}\mathrm{SE}^{1 / 4} \mathrm{sec} .18, \mathrm{~T} .8 \mathrm{~S} ., \mathrm{R} .3 \mathrm{E} \text {. } \\
\text { Unnamed tributary at mouth, }\end{array}$ & 27.4 & 18.0 & 21.4 & 738 & 969 & 10.8 \\
\hline & $\begin{array}{l}\mathrm{SE} 1 / 4 \text { sec. } 18, \text { T. } 8 \text { S., R. } 3 \mathrm{E} \text {. } \\
\text { Black Creek at unnamed tributary }\end{array}$ & 1.11 & 0 & 2.1 & 738 & 798 & 28.6 \\
\hline 289 . & $\begin{array}{l}\text { Black Creek at unnamed tributary, } \\
\text { SE1/4 sec. } 18, \text { T. } 8 \text { S., R. } 3 \text { E. } \\
\text { Black Creek at Sand Creek Highway }\end{array}$ & 28.5 & $18 . \dot{0}$ & 21.4 & 738 & 969 & 10.8 \\
\hline $\begin{array}{l}289 . \\
290 .\end{array}$ & $\begin{array}{l}\text { Black Creek at Sand Creek Highway, } \\
\text { SE1/4 sec. } 18, \text { T. } 8 \text { S., R. } 3 \text { E. } \\
\text { Black Creek above Weston Drain, }\end{array}$ & 28.6 & 17.6 & 21.8 & 736 & 969 & 10.7 \\
\hline $\begin{array}{l}291 . \\
292 .\end{array}$ & $\begin{array}{l}\text { Weston Drain at mouth, } \\
\text { SW1/4 sec. } 17, \text { T. } 8 \text { S., R. } 3 \text { E. } \\
\text { Black Creek at Weston Drain, }\end{array}$ & 1.61 & 0 & 2.2 & 733 & 770 & 16.8 \\
\hline 293. & $\begin{array}{l}\text { SW1 } 14 \text { sec. } 17, \text { T. } 8 \text { S., R. } 3 \text { E. } \\
\text { Black Creek above Baker and May Drain, }\end{array}$ & 30.5 & 17.0 & 22.4 & 733 & 969 & 10.5 \\
\hline 294. & $\begin{array}{l}\text { NE } 1 / 4 \text { sec. } 17, \text { T. } 8 \text { S., R. } 3 \text { E. } \\
\text { Baker and May Drain above unnamed tributary, }\end{array}$ & 31.4 & 16.1 & 23.3 & 729 & 969 & 10.3 \\
\hline 295. & $\begin{array}{l}\mathrm{SE}^{1 / 4} \text { sec. } 4, \text { T. } 8 \text { S., R. } 3 \mathrm{E} \text {. } \\
\text { Unnamed tributary at mouth. }\end{array}$ & 3.69 & 2.2 & 3.8 & 748 & 797 & 12.9 \\
\hline & SE $1 / 4$ sec. 4 , T. 8 S., R. 3 E. & 1.14 & 0 & 1.9 & 748 & 795 & 24.7 \\
\hline
\end{tabular}


PHYSIOGRAPHIC DATA FOR SELECTED SUBBASINS IN THE RIVER RAISIN BASIN - Continu»d

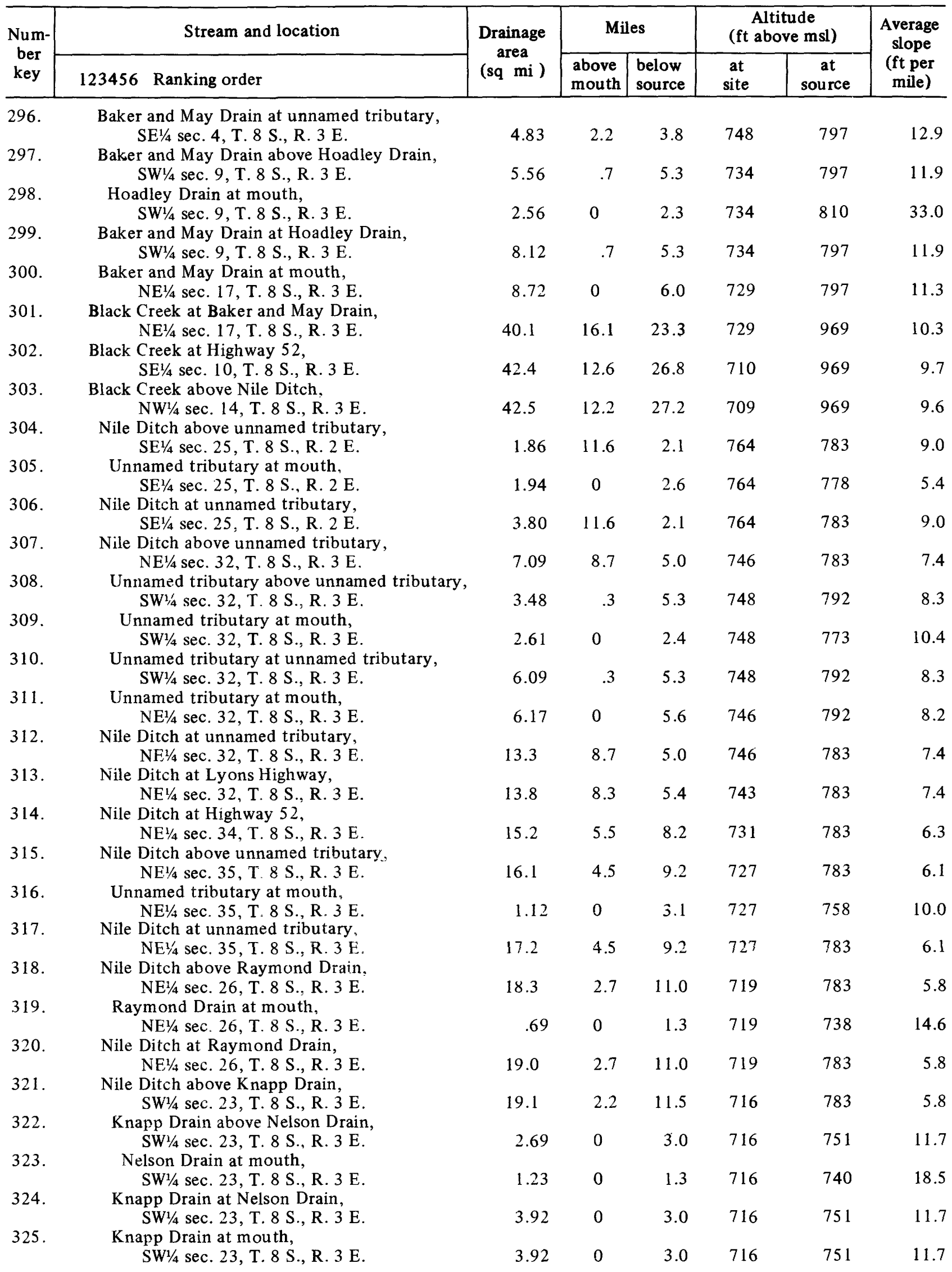


PHYSIOGRAPHIC DATA FOR SELECTED SUBBASINS IN THE RIVER RAISIN BASIN -Contir'גed

\begin{tabular}{|c|c|c|c|c|c|c|c|}
\hline \multirow{2}{*}{$\begin{array}{l}\text { Num- } \\
\text { ber } \\
\text { key }\end{array}$} & Stream and location & \multirow{2}{*}{$\begin{array}{l}\text { Drainage } \\
\text { area } \\
\text { (sq } \mathrm{mi} \text { ) }\end{array}$} & \multicolumn{2}{|c|}{ Miles } & \multicolumn{2}{|c|}{$\begin{array}{c}\text { Altitude } \\
\text { (ft above msl) }\end{array}$} & \multirow{2}{*}{$\begin{array}{c}\text { Average } \\
\text { slope } \\
\text { (ft per } \\
\text { mile) }\end{array}$} \\
\hline & 123456 Ranking order & & $\begin{array}{l}\text { above } \\
\text { mouth }\end{array}$ & $\begin{array}{l}\text { below } \\
\text { source }\end{array}$ & $\begin{array}{c}\text { at } \\
\text { site }\end{array}$ & $\begin{array}{c}\text { at } \\
\text { source }\end{array}$ & \\
\hline 326. & $\begin{array}{l}\text { Nile Ditch at Knapp Drain, } \\
\text { SW1/4 sec. } 23 \text {, T. } 8 \text { S., R. } 3 \text { E. }\end{array}$ & 23.0 & 2.2 & 11.5 & 716 & 783 & 5.8 \\
\hline 327. & $\begin{array}{l}\text { Nile Ditch above Abbott Drain, } \\
\text { SW1/4 sec. 14, T. } 8 \text { S., R. } 3 \text { E. }\end{array}$ & 23.8 & .7 & 13.0 & 711 & 783 & 5.5 \\
\hline 328. & $\begin{array}{l}\text { Abbott Drain at mouth, } \\
\text { SW } 1 / 4 \text { sec. } 14 \text {, T. } 8 \text { S., R. } 3 \text { E. }\end{array}$ & 1.10 & 0 & 2.5 & 711 & 760 & 19.6 \\
\hline 329. & $\begin{array}{l}\text { Nile Ditch at Abbott Drain, } \\
\text { SW1/4 sec. } 14 \text {, T. } 8 \text { S., R. } 3 \text { E. }\end{array}$ & 24.9 & .7 & 13.0 & 711 & 783 & 5.5 \\
\hline 330. & $\begin{array}{l}\text { Nile Ditch at Arnold Highway, } \\
\text { SW1/4 sec. 14, T. } 8 \text { S., R. } 3 \text { E. }\end{array}$ & 25.0 & .6 & 13.1 & 711 & 783 & 5.5 \\
\hline 331. & $\begin{array}{l}\text { Nile Ditch at mouth, } \\
\text { NW } 1 / 4 \text { sec. } 14 \text {, T. } 8 \text { S., R. } 3 \text { E. }\end{array}$ & 25.4 & 0 & 13.7 & 709 & 783 & 5.4 \\
\hline 332. & $\begin{array}{l}\text { Black Creek at Nile Ditch, } \\
\text { NW1/4 sec. } 14 \text {, T. } 8 \text { S., R. } 3 \text { E. }\end{array}$ & 67.9 & 12.2 & 27.2 & 709 & 969 & 9.6 \\
\hline 333. & $\begin{array}{l}\text { Black Creek above Walker Drain, } \\
\text { SW1/4 sec. } 11 \text {, T. } 8 \text { S., R. } 3 \text { E. } \\
\text { Walker Drain at mouth, }\end{array}$ & 68.0 & 11.8 & 27.6 & 708 & 969 & 9.4 \\
\hline 335. & $\begin{array}{l}\text { SW1/4 sec. 11, T. } 8 \text { S., R. } 3 \text { E. } \\
\text { Black Creek at Walker Drain, }\end{array}$ & 2.00 & 0 & 2.5 & 708 & 767 & 23.6 \\
\hline 336 & $\begin{array}{l}\text { SW1/4 sec. 11, T. } 8 \text { S., R. } 3 \text { E. } \\
\text { Black Creek above Holmes Drain, }\end{array}$ & 70.0 & 11.8 & 27.6 & 708 & 969 & 9.4 \\
\hline 337. & $\begin{array}{l}\text { SW1/4 sec. } 12 \text {, T. } 8 \text { S., R. } 3 \text { E. } \\
\text { Holmes Drain at mouth, }\end{array}$ & 70.9 & 10.2 & 29.2 & 706 & 969 & 9.4 \\
\hline 338. & $\begin{array}{l}\text { SW } 1 / 4 \text { sec. } 12, \text { T. } 8 \text { S., R. } 3 \text { E. } \\
\text { Black Creek at Holmes Drain, }\end{array}$ & 1.38 & 0 & 2.6 & 706 & 761 & 21.2 \\
\hline 339. & $\begin{array}{l}\mathrm{SW}^{1} 1 / 4 \text { sec. } 12, \text { T. } 8 \text { S., R. } 3 \text { E. } \\
\text { Black Creek above Foot Drain, }\end{array}$ & 72.3 & 10.2 & 29.2 & 706 & 969 & 9.4 \\
\hline 340. & $\begin{array}{l}\text { SE1/4 sec. } 6, \text { T. } 8 \text { S., R. } 4 \text { E. } \\
\text { Foot Drain at mouth, }\end{array}$ & 74.7 & 6.1 & 33.3 & 691 & 696 & 8.3 \\
\hline 341. & $\begin{array}{l}\mathrm{SE}^{1 / 4} \mathrm{sec} .6 \text {, T. } 8 \text { S., R. } 4 \text { E. } \\
\text { Black Creek at Foot Drain, }\end{array}$ & 2.58 & 0 & 2.6 & 691 & 730 & 15.0 \\
\hline 342 . & $\begin{array}{l}\text { SE1/4 sec. } 6, \text { T. } 8 \text { S., R. } 4 \text { E. } \\
\text { Black Creek above Walker Brook, }\end{array}$ & 77.3 & 6.1 & 33.3 & 691 & 969 & 8.3 \\
\hline 343. & $\begin{array}{l}\mathrm{SE}^{1 / 4} / \mathrm{sec} .5, \mathrm{~T} .8 \mathrm{~S} ., \mathrm{R} .4 \mathrm{E} \text {. } \\
\text { Walker Brook at mouth, }\end{array}$ & 77.6 & 5.4 & 34.0 & 689 & 969 & 8.2 \\
\hline 344. & $\begin{array}{l}\text { SE1/4 sec. 5, T. } 8 \text { S., R. } 4 \text { E. } \\
\text { Black Creek at Walker Brook, }\end{array}$ & 4.36 & 0 & 4.0 & 689 & 720 & 7.8 \\
\hline & $\begin{array}{l}\mathrm{SE}^{1 / 4} \text { sec. } 5, \text { T. } 8 \text { S., R. } 4 \text { E. } \\
\text { Black Creek above Gleason Brook, }\end{array}$ & 81.9 & 5.4 & 34.0 & 689 & 969 & 8.2 \\
\hline 345 . & $\begin{array}{l}\text { Black Creek above Gleason Brook, } \\
\text { NW1/4 sec. 4, T. } 8 \text { S., R. } 4 \text { E. } \\
\text { Gleason Brook at mouth. }\end{array}$ & 82.2 & 4.3 & 35.1 & 686 & 969 & 8.1 \\
\hline 346. & $\begin{array}{l}\text { Gleason Brook at mouth, } \\
\mathrm{NW}^{1 / 4} \mathrm{sec} .4 \text {, T. } 8 \mathrm{~S} ., \text { R. } 4 \text { E. }\end{array}$ & 4.93 & 0 & 6.0 & 686 & 785 & 16.5 \\
\hline 347. & $\begin{array}{l}\text { Black Creek at Gleason Brook, } \\
\text { NW1/4 sec. } 4 \text {, T. } 8 \text { S., R. } 4 \text { E. } \\
\text { Black Creek above Bear Creek, }\end{array}$ & 87.1 & 4.3 & 35.1 & 686 & 969 & 8.1 \\
\hline 348. & $\begin{array}{l}\text { Black Creek above Bear Creek, } \\
\text { SW1/4 sec. 4, T. } 8 \text { S., R. } 4 \text { E. }\end{array}$ & 87.2 & 3.9 & 35.5 & 685 & 969 & 8.0 \\
\hline 349. & $\begin{array}{l}\text { Bear Creek above unnamed tributary, } \\
\text { NW1/4 sec. } 20 \text {, T. } 9 \text { S., R. } 3 \text { E. }\end{array}$ & 4.81 & 16.6 & 5.2 & 756 & 797 & 7.9 \\
\hline 350. & $\begin{array}{l}\text { Unnamed tributary at mouth, } \\
\text { NW1/4 sec. } 20 \text {, T. } 9 \text { S., R. } 3 \text { E. }\end{array}$ & 6.40 & 0 & 5.7 & 756 & 790 & 6.0 \\
\hline 351. & $\begin{array}{l}\text { Bear Creek at unnamed tributary, } \\
\mathrm{NW}^{1} / 4 \mathrm{sec} .20, \mathrm{~T} .9 \mathrm{~S} ., \mathrm{R} .3 \mathrm{E} \text {. }\end{array}$ & 11.2 & 16.6 & 5.2 & 756 & 797 & 7.9 \\
\hline 352. & $\begin{array}{l}\text { Bear Creek above unnamed tributary, } \\
\text { NE1/4 sec. } 20 \text {, T. } 9 \mathrm{~S} ., \mathrm{R} .3 \mathrm{E} . \\
\text { Unnamed tributary at mouth. }\end{array}$ & 11.4 & 16.0 & 5.8 & 753 & 797 & 7.6 \\
\hline 353. & 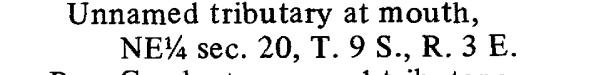 & 2.30 & 0 & 3.1 & 753 & 770 & 5.5 \\
\hline 354. & $\begin{array}{l}\text { Bear Creek at unnamed tributary, } \\
\text { NE } 1 / 4 \text { sec. } 20 \text {, T. } 9 \text { S., R. } 3 \text { E. } \\
\text { Rear }\end{array}$ & 13.7 & 16.0 & 5.8 & 753 & 757 & 7.6 \\
\hline 355. & $\begin{array}{l}\text { Bear Creek at Highway } 120, \\
\text { NE1/4 sec. } 15, \text { T. } 9 \text { S., R. } 3 \text { E. }\end{array}$ & 16.0 & 12.8 & 9.0 & 741 & 757 & 6.2 \\
\hline
\end{tabular}


PHYSIOGRAPHIC DATA FOR SELECTED SUBBASINS IN THE RIVER RAISIN BASIN - Continued

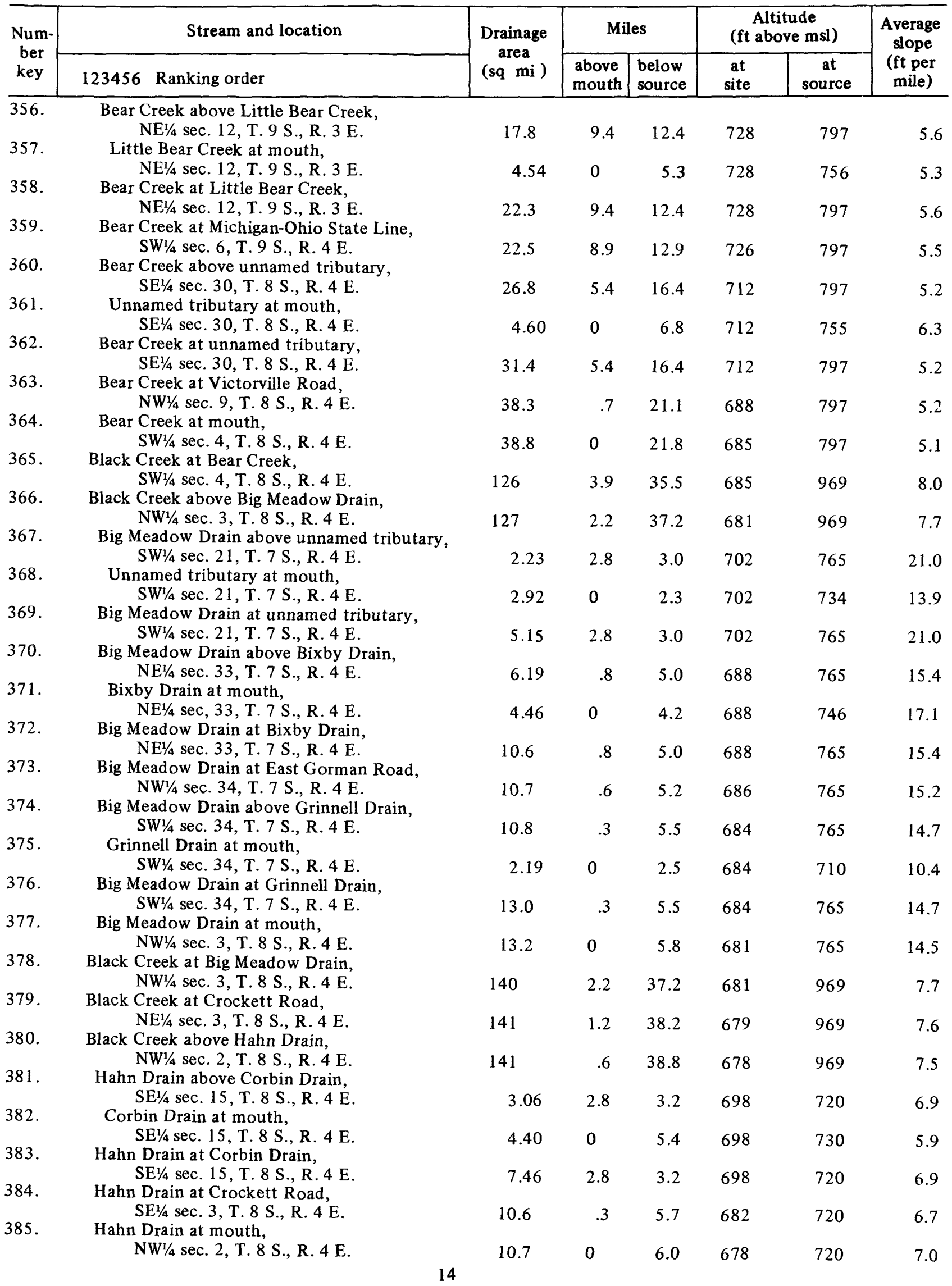


PHYSIOGRAPHIC DATA FOR SELECTED SUBBASINS IN THE RIVER RAISIN BASIN - Continued

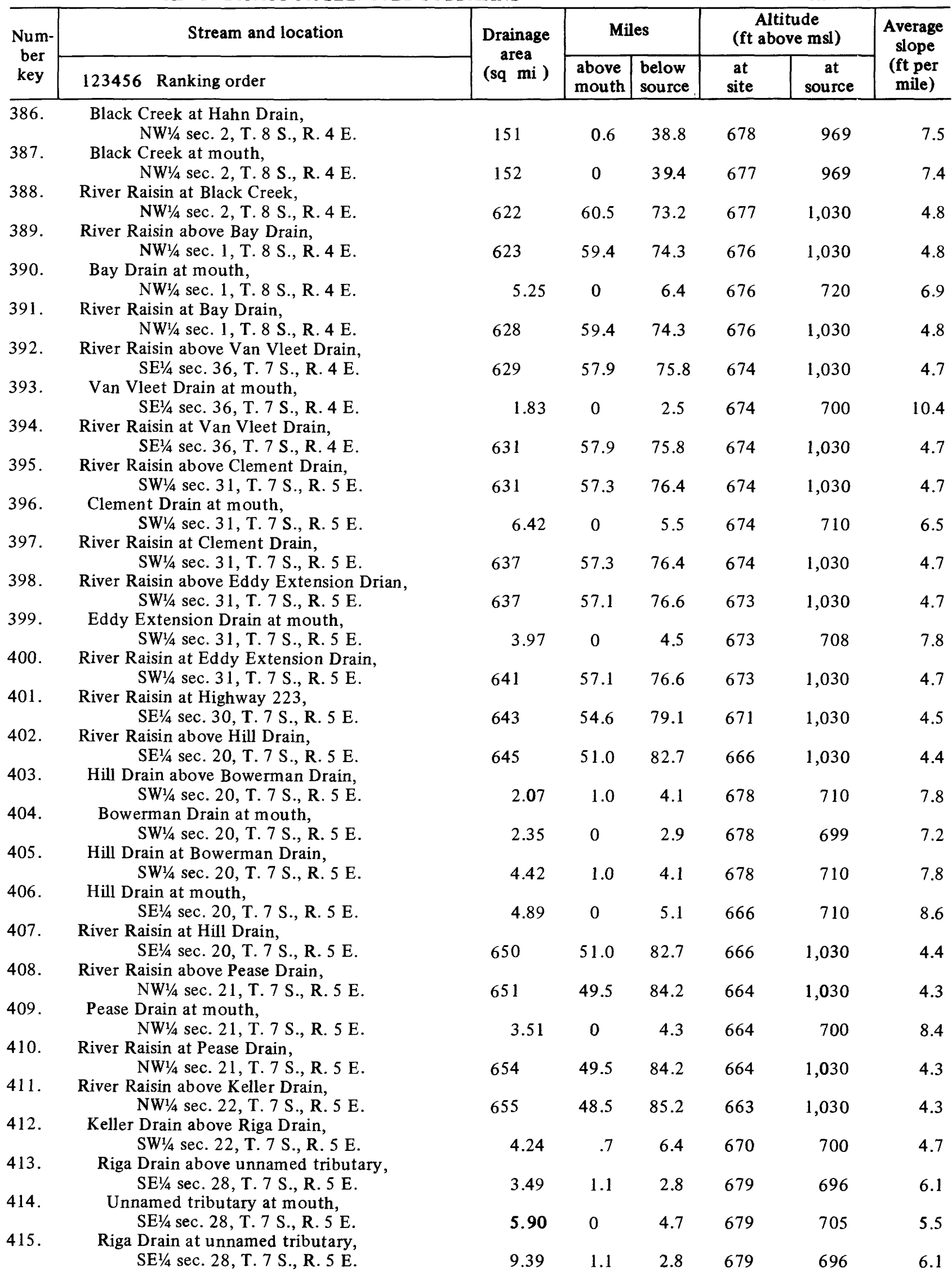


PHYSIOGRAPHIC DATA FOR SELECTED SUBBASINS IN THE RIVER RAISIN BASIN -Continued

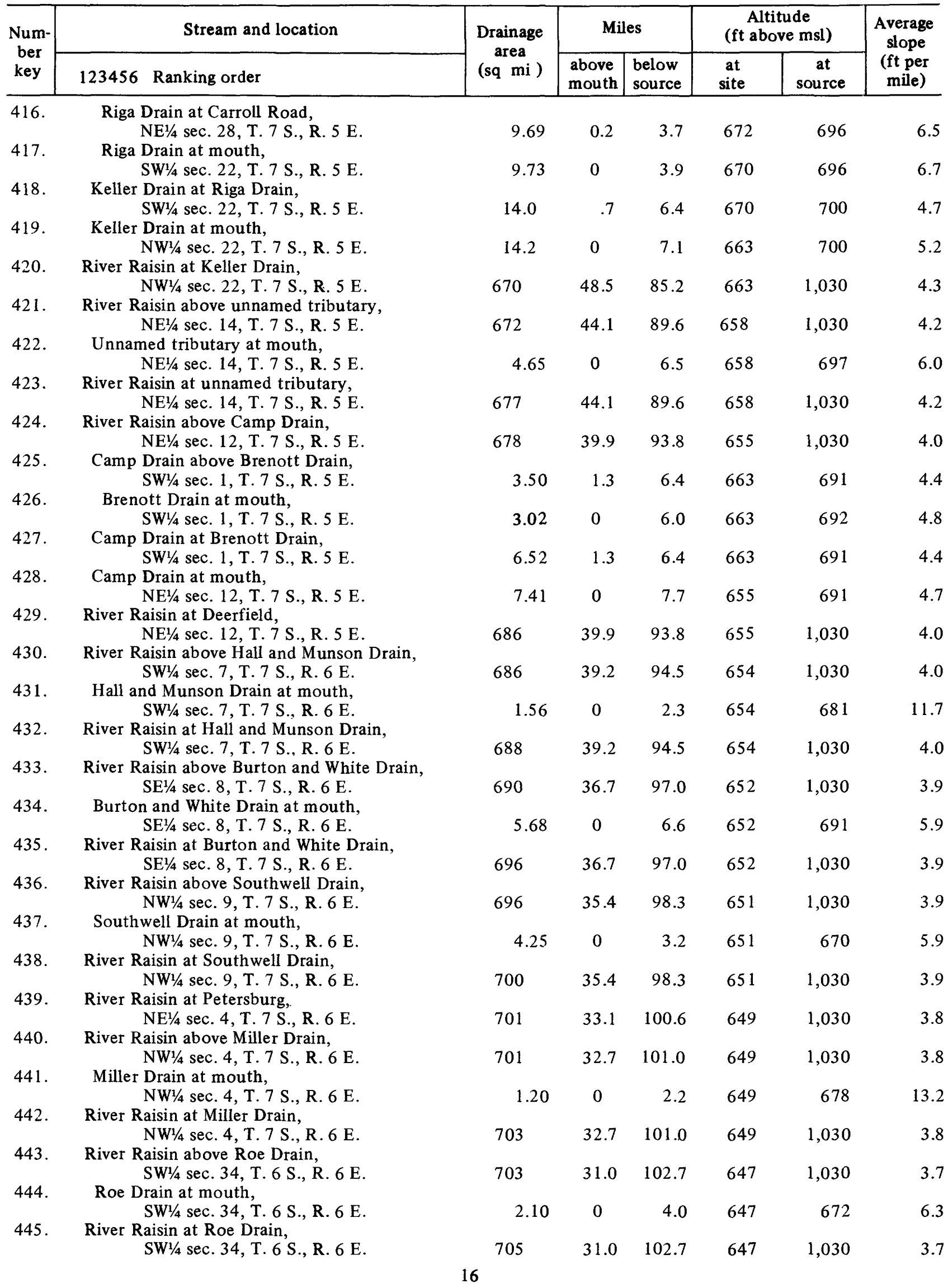


PHYSIOGRAPHIC DATA FOR SELECTED SUBBASINS IN THE RIVER RAISIN BASIN - Continued

\begin{tabular}{|c|c|c|c|c|c|c|c|}
\hline \multirow{2}{*}{$\begin{array}{c}\text { Num- } \\
\text { ber } \\
\text { key }\end{array}$} & Stream and location & \multirow{2}{*}{$\begin{array}{l}\text { Drainage } \\
\text { area } \\
\text { (sq } \mathrm{mi})\end{array}$} & \multicolumn{2}{|c|}{ Miles } & \multicolumn{2}{|c|}{$\begin{array}{c}\text { Altitude } \\
\text { (ft above msl) }\end{array}$} & \multirow{2}{*}{$\begin{array}{c}\text { Average } \\
\text { slope } \\
\text { (ft per } \\
\text { mile) }\end{array}$} \\
\hline & 123456 Ranking order & & $\begin{array}{l}\text { above } \\
\text { mouth }\end{array}$ & $\begin{array}{l}\text { below } \\
\text { source }\end{array}$ & $\begin{array}{c}\text { at } \\
\text { site }\end{array}$ & $\begin{array}{c}\text { at } \\
\text { source }\end{array}$ & \\
\hline $\begin{array}{l}446 . \\
447 .\end{array}$ & $\begin{array}{l}\text { River Raisin above Stacy Drain, } \\
\text { NE1/4 sec. } 35 \text {, T. } 6 \text { S., R. } 6 \text { E. } \\
\text { Stacy Drain at mouth, }\end{array}$ & 707 & 28.5 & 105.2 & 645 & 1,030 & 3.7 \\
\hline 448. & $\begin{array}{l}\text { NE } 1 / 4 \mathrm{sec} .35 \text {, T. } 6 \mathrm{~S} ., \text { R. } 6 \text { E. } \\
\text { River Raisin at Stacy Drain, }\end{array}$ & 3.89 & 0 & 4.7 & 645 & 680 & 7.4 \\
\hline \multirow[t]{2}{*}{449.} & $\begin{array}{l}\text { NE } 1 / 4 \text { sec. } 35 \text {, T. } 6 \text { S., R. } 6 \text { E. } \\
\text { River Raisin above Dunlap Drain, }\end{array}$ & 711 & 28.5 & 105.2 & 645 & 1,030 & 3.7 \\
\hline & $\begin{array}{l}\text { SE } 1 / 4 \text { sec. } 23 \text {, T. } 6 \text { S., R. } 6 \text { E. } \\
\text { Dunlap Drain at mouth, }\end{array}$ & 713 & 24.9 & 108.8 & 642 & 1,030 & 3.6 \\
\hline 451. & $\begin{array}{l}\text { SE } 1 / 4 \mathrm{sec} .23 \text {, T. } 6 \text { S., R. } 6 \text { E. } \\
\text { River Raisin at Dunlap Drain, }\end{array}$ & 3.55 & 0 & 5.6 & 642 & 672 & 5.4 \\
\hline 452. & $\begin{array}{l}\text { SE } 1 / 4 \mathrm{sec} .23, \mathrm{~T} .6 \mathrm{~S} ., \text { R. } 6 \text { E. } \\
\text { River Raisin above Little River Raisin, }\end{array}$ & 717 & 24.9 & 108.8 & 642 & 1,030 & 3.6 \\
\hline 453. & $\begin{array}{l}\text { SE } 1 / 4 \text { sec. 23, T. } 6 \text { S., R. } 6 \text { E. } \\
\text { Little River Raisin at Forche Road, }\end{array}$ & 717 & 24.7 & 109.0 & 642 & 1,030 & 3.6 \\
\hline 454. & $\begin{array}{l}\text { NE1/4 Sec. 7, T. } 7 \text { S., R. } 5 \text { E. } \\
\text { Little River Raisin at Lenawee Road, }\end{array}$ & 3.18 & 11.9 & 5.2 & 685 & 717 & 6.2 \\
\hline 455 . & $\begin{array}{l}\text { NE1/4 sec. 30, T. } 6 \mathrm{~S} ., \text { R. } 5 \text { E. } \\
\text { Little River Raisin above Swamp Raisin Creek, }\end{array}$ & 7.49 & 5.8 & 11.3 & 666 & 717 & 4.5 \\
\hline 456. & $\begin{array}{l}\text { SW1/4 sec. 22, T. } 6 \text { S., R. } 6 \text { E. } \\
\text { Swamp Raisin Creek above Westgate Drain, }\end{array}$ & 10.0 & 1.6 & 15.5 & 652 & 717 & 4.2 \\
\hline 457. & $\begin{array}{l}\mathrm{NW} 1 / 4 \mathrm{sec} .2 \text {, T. } 7 \mathrm{~S} ., \text { R. } 4 \mathrm{E} . \\
\text { Westgate Drain at mouth, }\end{array}$ & 3.99 & 12.5 & 3.0 & 697 & 738 & 13.7 \\
\hline 458. & $\begin{array}{l}\text { NW1/4 sec. 2, T. } 7 \text { S., R. } 4 \text { E. } \\
\text { Swamp Raisin Creek at Westgate Drain, }\end{array}$ & 2.13 & 0 & 2.6 & 697 & 750 & 20.4 \\
\hline \multirow[t]{2}{*}{459.} & $\begin{array}{l}\text { NW1/4 sec. 2, T. } 7 \text { S., R. } 4 \text { E. } \\
\text { Swamp Raisin Creek above Ash Drain, }\end{array}$ & 6.12 & 12.5 & 3.0 & 697 & 738 & 13.7 \\
\hline & $\begin{array}{l}\text { SW1/4 sec. } 35 \text {, T. } 6 \text { S., R. } 4 \text { E. } \\
\text { Ash Drain at mouth, }\end{array}$ & 6.23 & 12.2 & 3.3 & 695 & 738 & 13.0 \\
\hline 460 & $\begin{array}{l}\mathrm{SW} 1 / 4 \text { sec. } 35, \text { T. } 6 \mathrm{~S} ., \text { R. } 4 \mathrm{E} . \\
\text { Swamp Raisin Creek at Ash Drain, }\end{array}$ & 2.39 & 0 & 2.7 & 695 & 755 & 22.2 \\
\hline 461. & $\begin{array}{l}\mathrm{SW} 1 / 4 \mathrm{sec} .35, \mathrm{~T} .6 \mathrm{~S} ., \mathrm{R} .4 \mathrm{E} \text {. } \\
\text { Swamp Raisin Creek above unnamed tributary, }\end{array}$ & 8.62 & 12.2 & 3.3 & 695 & 738 & 13.0 \\
\hline 462. & $\begin{array}{l}\mathrm{SW} 1 / 4 \mathrm{sec} .32, \mathrm{~T} .6 \mathrm{~S} ., \mathrm{R} .5 \mathrm{E} \text {. } \\
\text { Unnamed tributary at mouth, }\end{array}$ & 10.8 & 9.2 & 6.3 & 678 & 738 & 9.5 \\
\hline 464. & $\begin{array}{l}\text { SW1/4 sec. 32, T. } 6 \text { S., R. } 5 \text { E. } \\
\text { Swamp Raisin Creek at unnamed tributary, }\end{array}$ & 1.40 & 0 & 3.5 & 678 & 716 & 10.8 \\
\hline 465. & $\begin{array}{l}\text { SW1/4 sec. 32, T. } 6 \text { S., R. } 5 \text { E. } \\
\text { Swamp Raisin Creek above Isley Drain, }\end{array}$ & 12.2 & 9.2 & 6.3 & 678 & 738 & 9.5 \\
\hline 466. & $\begin{array}{l}\mathrm{SE}^{1 / 4} / 4 \mathrm{sec} .32, \text { T. } 6 \text { S., R. } 5 \text { E. } \\
\text { Isley Drain at mouth, }\end{array}$ & 13.1 & 8.4 & 7.1 & 676 & 738 & 8.7 \\
\hline 467. & $\begin{array}{l}\text { SE1/4 sec. } 32 \text {, T. } 6 \text { S., R. } 5 \text { E. } \\
\text { Swamp Raisin Creek at Isley Drain, }\end{array}$ & 2.46 & 0 & 4.7 & 676 & 708 & 6.8 \\
\hline 468. & $\begin{array}{l}\text { SE } 1 / 4 \text { sec. } 32 \text {, T. } 6 \text { S., R. } 5 \text { E. } \\
\text { Swamp Raisin Creek above Spring Brook, }\end{array}$ & 15.6 & 8.4 & 7.1 & 676 & 738 & 8.7 \\
\hline 469. & $\begin{array}{l}\text { NW11/4 sec. } 34, \text { T. } 6 \text { S., R. } 5 \text { E. } \\
\text { Spring Brook above Woodruff Brook, }\end{array}$ & 16.2 & 7.0 & 8.5 & 671 & 738 & 7.9 \\
\hline 470 & $\begin{array}{l}\text { NE } 1 / 4 \text { sec. } 32, \text { T. } 6 \text { S., R. } 5 \text { E. } \\
\text { Woodruff Brook at mouth, }\end{array}$ & 1.94 & 1.5 & 3.5 & 678 & 728 & 14.3 \\
\hline 471 & $\begin{array}{l}\mathrm{NE}^{1 / 4} / 4 \mathrm{sec} .32 \text {, T. } 6 \mathrm{~S} ., \mathrm{R} .5 \mathrm{E} \text {. } \\
\text { Spring Brook at Woodruff Brook, }\end{array}$ & 3.66 & 0 & 4.4 & 678 & 770 & 20.9 \\
\hline 472. & $\begin{array}{l}\mathrm{NE} 1 / 4 \text { sec. } 32, \mathrm{~T} .6 \mathrm{~S} ., \text { R. } 5 \mathrm{E} \text {. } \\
\text { Spring Brook at mouth, }\end{array}$ & 5.60 & 1.5 & 3.5 & 678 & 728 & 14.3 \\
\hline 473. & $\begin{array}{c}\text { NW1/4 sec. } 34, \text { T. } 6 \text { S., R. } 5 \text { E. } \\
\text { Swamp Raisin Creek at Spring Brook, }\end{array}$ & 6.19 & 0 & 5.0 & 671 & 728 & 11.4 \\
\hline 474 & $\begin{array}{l}\text { NW1/4 sec. } 34, \text { T. } 6 \text { S., R. } 5 \text { E. } \\
\text { Swamp Raisin Creek above Schwab Drain, }\end{array}$ & 22.4 & 7.0 & 8.5 & 671 & 738 & 7.9 \\
\hline 475 & $\begin{array}{l}\text { SE } 1 / 4 \text { sec. } 27, \text { T. } 6 \text { S., R. } 5 \text { E. } \\
\text { Schwab Drain at mouth, }\end{array}$ & 23.7 & 6.3 & 9.2 & 669 & 738 & 7.5 \\
\hline & SE $1 / 4$ sec. 27, T. 6 S., R. 5 E. & 3.32 & 0 & 5.9 & 669 & 768 & 16.8 \\
\hline
\end{tabular}


PHYSIOGRAPHIC DATA FOR SELECTED SUBBASINS IN THE RIVER RAISIN BASIN - Continued

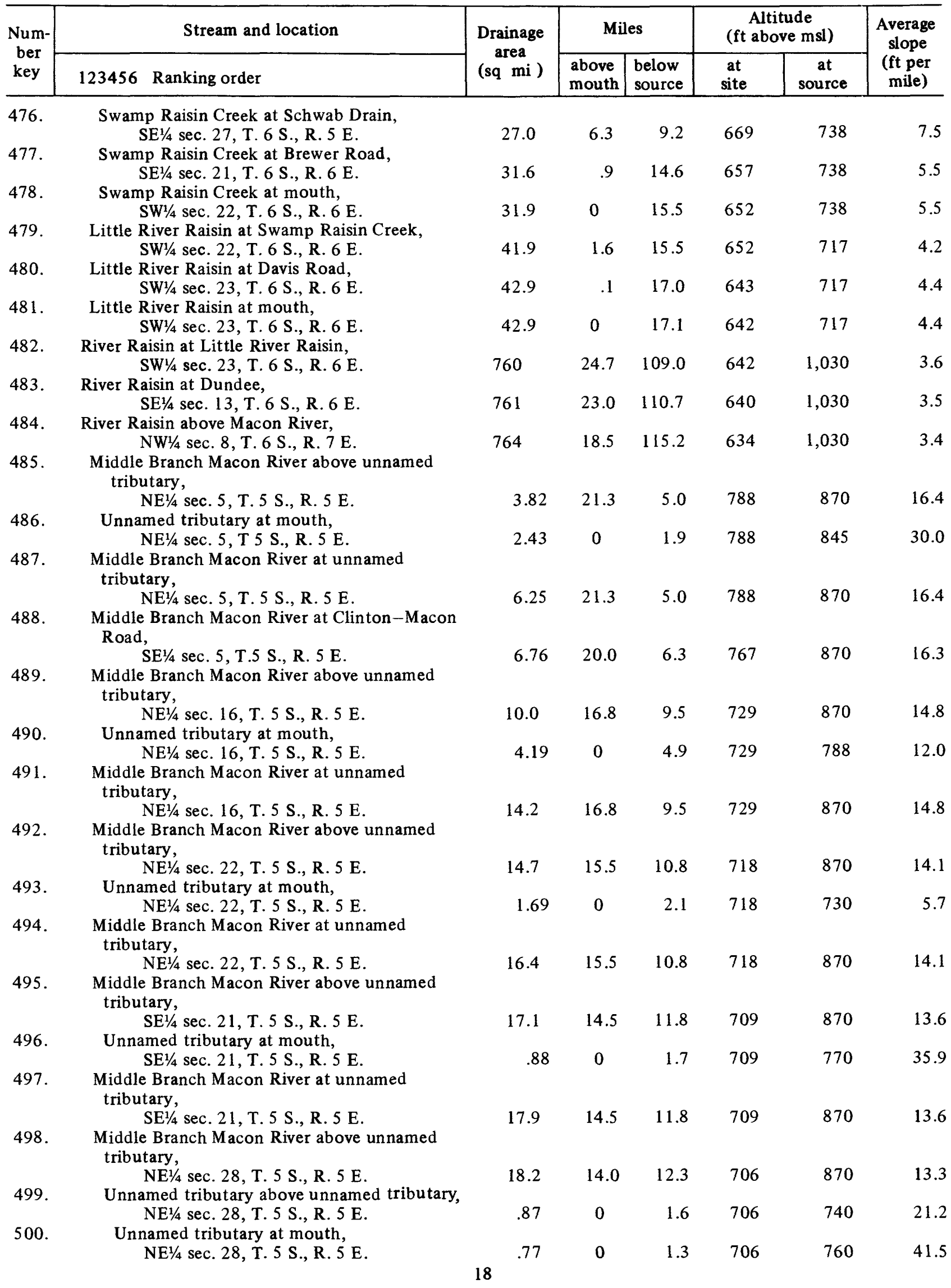


PHYSIOGRAPHIC DATA FOR SELECTED SUBBASINS IN THE RIVER RAISIN BASIN - Continued

\begin{tabular}{|c|c|c|c|c|c|c|c|}
\hline \multirow{2}{*}{$\begin{array}{c}\text { Num- } \\
\text { ber } \\
\text { key }\end{array}$} & Stream and location & \multirow{2}{*}{$\begin{array}{l}\text { Drainage } \\
\text { area } \\
\text { (sq } \mathrm{mi})\end{array}$} & \multicolumn{2}{|c|}{ Miles } & \multicolumn{2}{|c|}{$\begin{array}{c}\text { Altitude } \\
\text { (ft above msl) }\end{array}$} & \multirow{2}{*}{$\begin{array}{c}\text { Average } \\
\text { slope } \\
\text { (ft per } \\
\text { mile) }\end{array}$} \\
\hline & 123456 Ranking order & & $\begin{array}{l}\text { above } \\
\text { mouth }\end{array}$ & $\begin{array}{l}\text { below } \\
\text { source }\end{array}$ & $\begin{array}{c}\text { at } \\
\text { site }\end{array}$ & $\begin{array}{c}\text { at } \\
\text { source }\end{array}$ & \\
\hline 501. & $\begin{array}{l}\text { Unnamed tributary at unnamed tributary, } \\
\text { NE1/4 sec. } 28 \text {, T. } 5 \text { S., R. } 5 \text { E. }\end{array}$ & 1.64 & 0 & 1.6 & 706 & 740 & 21.2 \\
\hline 502. & $\begin{array}{l}\text { Middle Branch Macon River at Ridge Highway, } \\
\text { NE1/4 sec. } 28 \text {, T. } 5 \text { S., R. } 5 \text { E. }\end{array}$ & 19.8 & 14.0 & 12.3 & 706 & 870 & 13.3 \\
\hline 504. & $\begin{array}{l}\text { SW11/4 sec. } 36, \text { T. } 5 \text { S., R. } 5 \text { E. } \\
\text { Unnamed tributary at mouth. }\end{array}$ & 21.9 & 10.3 & 16.0 & 682 & 870 & 11.8 \\
\hline 505. & $\begin{array}{l}\text { Middle Branch Macon River at unnamed } \\
\text { tributary }\end{array}$ & 2.09 & 0 & 3.2 & 682 & 720 & 11.9 \\
\hline 506. & $\begin{array}{l}\mathrm{SW} 1 / 4 \text { sec. } 36, \mathrm{~T} .5 \mathrm{~S} ., \mathrm{R} .5 \mathrm{E} \text {. } \\
\text { Middle Branch Macon River above unnamed } \\
\text { tributary, }\end{array}$ & 24.0 & 10.3 & 16.0 & 682 & 870 & 11.8 \\
\hline \multirow[t]{2}{*}{507.} & \multirow{2}{*}{ 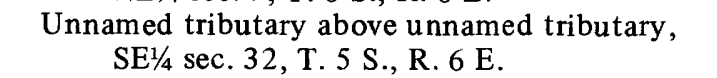 } & 25.4 & 7.1 & 19.2 & 661 & 870 & 10.9 \\
\hline & & 2.89 & .5 & 5.4 & 668 & 718 & 9.3 \\
\hline 508. & $\begin{array}{l}\text { Unnamed tributary above unnamed tributary, } \\
\text { NW1/4 sec. } 32 \text {, T. } 5 \text { S., R. } 6 \text { E. }\end{array}$ & 2.05 & 6 & 3.2 & 669 & 699 & 9.4 \\
\hline \multirow[t]{2}{*}{510.} & \multirow{2}{*}{$\begin{array}{l}\text { Unnamed tributary at unnamed tributary, } \\
\text { NW1/4 sec. } 32 \text {, T. } 5 \text { S., R. } 6 \text { E. }\end{array}$} & 1.07 & 0 & 2.8 & 669 & 700 & 11.1 \\
\hline & & 3.12 & 1.5 & 2.2 & 669 & 699 & 13.6 \\
\hline 511. & $\begin{array}{l}\text { Unnamed tributary at mouth, } \\
\mathrm{SE}^{1 / 4} \text { sec. } 32, \text { T. } 5 \text { S., R. } 6 \text { E. }\end{array}$ & 3.37 & 0 & 3.7 & 668 & 699 & 8.4 \\
\hline 513. & $\begin{array}{l}\text { Unnamed tributary at unnamed tributary, } \\
\mathrm{SE} 1 / 4 \mathrm{sec} .32 \text {, T. } 5 \mathrm{~S} \text {., R. } 6 \mathrm{E} \text {. }\end{array}$ & 6.26 & .5 & 5.4 & 668 & 718 & 9.3 \\
\hline 514. & $\begin{array}{l}\text { Unnamed tributary at mouth, } \\
\text { NE } 1 / 4 \text { sec. } 5, \text { T. } 6 \text { S., R. } 6 \text { E. } \\
\text { Middle Branch Macon River at unnamed }\end{array}$ & 6.37 & 0 & 5.4 & 661 & 718 & 10.6 \\
\hline 515. & $\begin{array}{l}\mathrm{NE} 1 / 4 \text { Sec. } 5 \text {, T. } 6 \text { S., R. } 6 \text { E. } \\
\text { Middle Branch Macon River above unnamed } \\
\text { tributary, }\end{array}$ & 31.8 & 7.1 & 19.2 & 661 & 870 & 10.9 \\
\hline 516. & $\begin{array}{c}\mathrm{NE}^{1 / 4} \text { sec. } 9, \text { T. } 6 \text { S., R. } 6 \mathrm{E} . \\
\text { Unnamed tributary at mouth, } \\
\text { NE}^{1 / 4} \text { sec. } 9, \text { T. } 6 \text { S., R. } 6 \text { E. }\end{array}$ & 35.0 & 5.6 & 20.7 & 654 & 870 & 10.4 \\
\hline 517. & $\begin{array}{l}\text { NE } 1 / 4 \text { sec. } 9 \text {, T. } 6 \text { S., R. } 6 \text { E. } \\
\text { Middle Branch Macon River at unnamed } \\
\text { tributary, }\end{array}$ & 7.15 & 0 & 5.7 & 654 & 684 & 5.3 \\
\hline 518. & $\begin{array}{l}\text { NE } 1 / 4 \text { sec. 9, T. } 6 \text { S., R. } 6 \text { E. } \\
\text { Middle Branch Macon River at Wilcox Road }\end{array}$ & 42.2 & 5.6 & 20.7 & 654 & 870 & 10.4 \\
\hline 519. & $\begin{array}{l}\mathrm{NW} 1 / 4 \mathrm{sec} .11 \text {, T. } 6 \text { S., R. } 6 \text { E. } \\
\text { Middle Branch Macon River above Leppleman } \\
\text { Drain, }\end{array}$ & 43.8 & 4.2 & 22.1 & 646 & 870 & 10.2 \\
\hline 520. & $\begin{array}{l}\text { SE } 1 / 4 \text { sec. } 2, \text { T. } 6 \text { S., R. } 6 \text { E. } \\
\text { Leppleman Drain at mouth, }\end{array}$ & 43.9 & 3.9 & 22.4 & 644 & 870 & 10.1 \\
\hline 521. & $\begin{array}{l}\mathrm{SE}^{1 / 4} \mathrm{sec} .2 \text {, T. } 6 \mathrm{~S} ., \mathrm{R} .6 \mathrm{E} \text {. } \\
\text { Middle Branch Macon River at Leppleman Drain, }\end{array}$ & 2.19 & 0 & 2.9 & 644 & 670 & 9.0 \\
\hline 522. & $\begin{array}{l}\mathrm{SE}^{1 / 4} \text { sec. } 2 \text {, T. } 6 \text { S., R. } 6 \text { E. } \\
\text { Middle Branch Macon River above South Branch } \\
\text { Macon River, }\end{array}$ & 46.1 & 3.9 & 22.4 & 644 & 870 & 10.1 \\
\hline 523. & $\begin{array}{l}\text { SE1/4 sec. 2, T. } 6 \text { S., R. } 6 \text { E. } \\
\text { South Branch Macon River at Macon Road, }\end{array}$ & 46.4 & 3.5 & 22.8 & 642 & 870 & 10.0 \\
\hline 524. & $\begin{array}{l}\text { NE } 1 / 4 \text { sec. } 24, \text { T. } 5 \text { S., R. } 4 \text { E. } \\
\text { South Branch Macon River above unnamed } \\
\text { tributary, }\end{array}$ & 2.49 & 17.5 & 2.1 & 798 & 840 & 20.0 \\
\hline 525. & $\begin{array}{l}\mathrm{SW}^{1} 1 / 4 \text { sec. } 19, \text { T. } 5 \mathrm{~S} ., \text { R. } 5 \mathrm{E} \text {. } \\
\text { Unnamed tributary at mouth, }\end{array}$ & 2.70 & 16.5 & 3.1 & 781 & 840 & 19.0 \\
\hline & SW1/4 sec. 19, T. 5 S., R. 5 E. & 1.76 & 0 & 2.2 & 781 & 815 & 15.5 \\
\hline
\end{tabular}


PHYSIOGRAPHIC DATA FOR SELECTED SUBBASINS IN THE RIVER RAISIN BASIN - Continued

\begin{tabular}{|c|c|c|c|c|c|c|c|}
\hline \multirow{2}{*}{$\begin{array}{c}\text { Num- } \\
\text { ber } \\
\text { key }\end{array}$} & Stream and location & \multirow{2}{*}{$\begin{array}{l}\text { Drainage } \\
\text { area } \\
\text { (sq } \mathrm{mi})\end{array}$} & \multicolumn{2}{|c|}{ Miles } & \multicolumn{2}{|c|}{$\begin{array}{c}\text { Altitude } \\
\text { (ft above msl) }\end{array}$} & \multirow{2}{*}{$\begin{array}{l}\text { Average } \\
\text { slope } \\
\text { (ft per } \\
\text { mile) }\end{array}$} \\
\hline & 123456 Ranking order & & $\begin{array}{l}\text { above } \\
\text { mouth }\end{array}$ & $\begin{array}{l}\text { below } \\
\text { source }\end{array}$ & $\begin{array}{l}\text { at } \\
\text { site }\end{array}$ & $\begin{array}{c}\text { at } \\
\text { source }\end{array}$ & \\
\hline 526. & $\begin{array}{l}\text { South Branch Macon River at innamed } \\
\text { tributary, } \\
\text { SW } 1 \frac{1}{4} \text { sec. } 19 \text {, T. } 5 \text { S., R. } 5 \text { E. }\end{array}$ & 4.46 & 16.5 & 3.1 & 781 & 840 & 19.0 \\
\hline 527. & $\begin{array}{l}\text { South Branch Macon River at Tecumseh Road, } \\
\text { SE } 1 / 4 \text { sec. } 31 \text {, T. } 5 \text { S., R. } 5 \text { E. }\end{array}$ & 7.88 & 13.4 & 6.2 & 740 & 840 & 16.1 \\
\hline 528. & $\begin{array}{l}\text { South Branch Macon River at Ridge Road, } \\
\text { SE } 1 / 4 \text { sec. } 6 \text {, T. } 6 \text { S., R. } 5 \text { E. }\end{array}$ & 9.17 & 11.9 & 7.7 & 719 & 840 & 15.7 \\
\hline 529. & $\begin{array}{l}\text { South Branch Macon River at Britton Road, } \\
\text { NE } 1 / 4 \text { sec. } 16 \text {, T. } 6 \text { S., R. } 5 \text { E. }\end{array}$ & 10.5 & 9.0 & 10.6 & 679 & 840 & 15.2 \\
\hline 531. & $\begin{array}{l}\text { South Branch Macon River above Dibble Drain, } 14 \text {, T. } 6 \text { S., R. } 5 \text { E. } \\
\text { Dibble Drain above Sutton Drain, }\end{array}$ & 11.1 & 7.4 & 12.2 & 673 & 840 & 13.7 \\
\hline 523 & SE $1 / 4$ sec. 16, T. 6 S., R. 5 E. & 1.58 & 1.6 & 3.8 & 681 & 770 & 23.4 \\
\hline 533 & SE $1 / 4$ sec. 16 , T. 6 S., R. 5 E. & 5.05 & 0 & 5.3 & 681 & 800 & 22.5 \\
\hline 534. & $\begin{array}{l}\text { Dibble Drain at Sutton Drain, } \\
\text { SE } 1 / 4 \text { sec. } 16, \text { T. } 6 \text { S., R. } 5 \text { E. } \\
\text { Dibble Drain at mouth, }\end{array}$ & 6.63 & 1.6 & 3.8 & 681 & 770 & 23.4 \\
\hline 535 & $\begin{array}{l}\text { SW } 1 / 4 \text { sec. } 14, \text { T. } 6 \text { S., R. } 5 \text { E. } \\
\text { South Branch }\end{array}$ & 7.02 & 0 & 5.4 & 673 & 770 & 18.0 \\
\hline 536. & $\begin{array}{l}\text { SW } 1 / 4 \text { sec. 14, T. } 6 \text { S., R. } 5 \text { E. } \\
\text { South Branch Macon River above Kelly Drain. }\end{array}$ & 18.1 & 7.4 & 12.2 & 673 & 840 & 13.7 \\
\hline 537. & $\begin{array}{l}\text { SE } 1 / 4 \text { sec. } 13, \text { T. } 6 \mathrm{~S} ., \text { R. } 5 \mathrm{E} . \\
\text { Kelly Drain above Wilson Drain. }\end{array}$ & 18.5 & 5.9 & 13.7 & 668 & 840 & 12.6 \\
\hline 538. & $\begin{array}{l}\text { SE } 1 / 4 \text { sec. } 23, \text { T. } 6 \text { S., R. } 5 \text { E. } \\
\text { Wilson Drain at mouth, }\end{array}$ & 4.58 & 1.3 & 7.2 & 671 & 780 & 15.1 \\
\hline 539. & $\begin{array}{l}\text { SE } 1 / 4 \text { sec. } 23, \text { T. } 6 \text { S., R. } 5 \text { E. } \\
\text { Kelly Drain at Wilson Drain, }\end{array}$ & 1.40 & 0 & 3.2 & 671 & 690 & 5.9 \\
\hline & SE $1 / 4$ sec. 23, T. 6 S., R. 5 E. & 5.98 & 1.3 & 7.2 & 671 & 780 & 15.1 \\
\hline 540. & $\begin{array}{l}\text { Kelly Drain above Rixon Drain, } \\
\text { SE } 1 / 4 \text { sec. } 13, \text { T. } 6 \text { S., R. } 5 \text { E. } \\
\text { Rixon Drain at mouth, }\end{array}$ & 6.70 & .4 & 8.1 & 669 & 780 & 13.7 \\
\hline 542. & $\begin{array}{l}\text { SE } 1 / 4 \text { sec. } 13, \text { T. } 6 \text { S., R. } 5 \text { E. } \\
\text { Kelly Drain at Rixon Drain, }\end{array}$ & 1.49 & 0 & 4.7 & 669 & 705 & 7.6 \\
\hline 543. & $\begin{array}{l}\text { SE } 1 / 4 \text { sec. } 13, \text { T. } 6 \text { S., R. } 5 \text { E. } \\
\text { Kelly Drain at mouth, }\end{array}$ & 8.19 & .4 & 8.1 & 669 & 780 & 13.7 \\
\hline 544 & $\begin{array}{l}\text { SE } 1 / 4 \text { sec. } 13, \text { T. } 6 \text { S., R. } 5 \text { E. } \\
\text { South }\end{array}$ & 8.25 & 0 & 8.5 & 668 & 780 & 13.2 \\
\hline 544. & $\begin{array}{l}\text { South Branch Macon River at Kelly Drain, } \\
\text { SE } 1 / 4 \text { sec. } 13 \text {, T. } 6 \text { S., R. } 5 \text { E. } \\
\text { South Branch Macon River above Schreeder } \\
\text { Brook, }\end{array}$ & 26.8 & 5.9 & 13.7 & 668 & 840 & 12.5 \\
\hline 546. & $\begin{array}{l}\text { SE } 1 / 4 \text { sec. } 13, \text { T. } 6 \text { S., R. } 5 \text { E. } \\
\text { Schreeder Brook at Ridge Road, }\end{array}$ & 26.8 & 5.8 & 13.8 & 668 & 840 & 12.5 \\
\hline 547. & $\begin{array}{l}\text { NE } 1 / 4 \text { sec. } 32, \text { T. } 5 \text { S., R. } 5 \text { E. } \\
\text { Schreeder Brook above Springbrook Drain, }\end{array}$ & 2.14 & 6.9 & 3.8 & 720 & 789 & 18.2 \\
\hline 548. & $\begin{array}{l}\text { SE } 1 / 4 \text { sec. } 10, \mathrm{~T} .6 \mathrm{~S} ., \mathrm{R} .5 \mathrm{E} . \\
\text { Springbrook Drain at mouth, }\end{array}$ & 5.47 & 2.8 & 7.9 & 679 & 789 & 13.9 \\
\hline 549. & $\begin{array}{l}\text { SE } 1 / 4 \text { sec. } 10, \text { T. } 6 \text { S., R. } 5 \text { E. } \\
\text { Schreeder Brook at Springbrook Drain, }\end{array}$ & 4.35 & 0 & 5.2 & 679 & 760 & 15.6 \\
\hline 550. & $\begin{array}{l}\mathrm{SE}^{1 / 4} \text { sec. } 10, \text { T. } 6 \mathrm{~S} ., \mathrm{R} .5 \mathrm{E} \text {. } \\
\text { Schreeder Brook at mouth, }\end{array}$ & 9.82 & 2.8 & 7.9 & 679 & 789 & 13.9 \\
\hline 551. & $\begin{array}{l}\text { SE } 1 / 4 \text { sec. } 13, \text { T. } 6 \text { S., R. } 5 \text { E. } \\
\text { South Branch Macon River at Schreeder Brook, }\end{array}$ & 11.3 & 0 & 10.7 & 668 & 789 & 11.3 \\
\hline 552. & $\begin{array}{l}\text { SE } 1 / 4 \text { sec. } 13, \text { T. } 6 \text { S., R. } 5 \text { E. } \\
\text { South Branch Macon River at Tecumseh Road, }\end{array}$ & 38.2 & 5.8 & 13.8 & 668 & 840 & 12.5 \\
\hline 553. & $\begin{array}{l}\text { NW1/4 Sec. 15, T. } 6 \text { S., R. } 6 \text { E. } \\
\text { South Branch Macon River at Wilcox Road, }\end{array}$ & 43.6 & 2.1 & 17.5 & 648 & 840 & 11.3 \\
\hline 554. & $\begin{array}{l}\text { NW1/4 sec. } 11, \text { T. } 6 \text { S., R. } 6 \text { E. } \\
\text { South Branch Macon River at mouth, }\end{array}$ & 45.5 & .8 & 18.8 & 646 & 840 & 10.3 \\
\hline & SE $1 / 4$ sec. 2, T. 6 S., R. 6 E. & 46.0 & 0 & 19.6 & 642 & 840 & 10.1 \\
\hline
\end{tabular}


PHYSIOGRAPHIC DATA FOR SELECTED SUBBASINS IN THE RIVER RAISIN BASIN - Continue

\begin{tabular}{|c|c|c|c|c|c|c|c|}
\hline \multirow{2}{*}{$\begin{array}{c}\text { Num- } \\
\text { ber } \\
\text { key }\end{array}$} & Stream and location & \multirow{2}{*}{$\begin{array}{l}\text { Drainage } \\
\text { area } \\
\text { (sq } \mathrm{mi})\end{array}$} & \multicolumn{2}{|c|}{ Miles } & \multicolumn{2}{|c|}{$\begin{array}{c}\text { Altitude } \\
\text { (ft above msl) }\end{array}$} & \multirow{2}{*}{$\begin{array}{c}\text { Average } \\
\text { slope } \\
\text { (ft per } \\
\text { mile) }\end{array}$} \\
\hline & 123456 Ranking order & & $\begin{array}{l}\text { above } \\
\text { mouth }\end{array}$ & $\begin{array}{l}\text { below } \\
\text { source }\end{array}$ & $\begin{array}{l}\text { at } \\
\text { site }\end{array}$ & $\begin{array}{c}\text { at } \\
\text { source }\end{array}$ & \\
\hline 555. & $\begin{array}{l}\text { Macon River at South Branch Macon River, } \\
\text { SE } 1 / 4 \text { sec. } 2 \text {, T. } 6 \text { S., R. } 6 \text { E. } \\
\text { Macon River above North Branch Macon River, }\end{array}$ & 92.4 & 3.5 & 22.8 & 642 & 870 & 9.8 \\
\hline 557. & & 94.3 & 2.4 & 23.9 & 639 & 870 & 9.7 \\
\hline 558. & $\begin{array}{l}\mathrm{SE}^{1 / 4} \mathrm{sec} .26, \mathrm{~T} .4 \mathrm{~S} ., \mathrm{R} .5 \mathrm{E} \text {. } \\
\text { North Branch Macon River above unnamed } \\
\text { tributary, }\end{array}$ & 7.55 & 16.6 & 4.0 & 746 & 817 & 17.8 \\
\hline 559. & $\begin{array}{l}\mathrm{NE}^{1 / 4} \mathrm{sec} .1, \mathrm{~T} .5 \mathrm{~S} ., \text { R. } 5 \mathrm{E} . \\
\text { Unnamed tributary at mouth, }\end{array}$ & 8.53 & 14.5 & 6.1 & 723 & 817 & 15.4 \\
\hline 560. & $\begin{array}{l}\text { NE } 1 / 4 \text { sec. 1, T. } 5 \text { S., R. } 5 \text { E. } \\
\text { North Branch Macon River at unnamed } \\
\text { tributary, }\end{array}$ & 1.62 & 0 & 2.7 & 723 & 770 & 17.4 \\
\hline 561. & $\begin{array}{l}\mathrm{NE}^{1 / 4} \mathrm{sec} .1, \mathrm{~T} .5 \mathrm{~S} ., \mathrm{R} .5 \mathrm{E} \text {. } \\
\text { North Branch Macon River above unnamed } \\
\text { tributary, }\end{array}$ & 10.2 & 14.5 & 6.1 & 723 & 817 & 15.4 \\
\hline 562. & $\begin{array}{l}\mathrm{SW}^{1} / 4 \mathrm{sec} .31, \mathrm{~T} .4 \mathrm{~S} ., \mathrm{R} .6 \mathrm{E} . \\
\text { Unnamed tributary at mouth, }\end{array}$ & 10.4 & 13.7 & 6.9 & 716 & 817 & 14.6 \\
\hline 563. & $\begin{array}{l}\mathrm{SW} 1 / 4 \mathrm{sec} .31, \mathrm{~T} .4 \mathrm{~S} ., \mathrm{R} .6 \mathrm{E} \text {. } \\
\text { North Branch Macon River at unnamed } \\
\text { tributary, }\end{array}$ & 3.06 & 0 & 3.1 & 716 & 779 & 20.3 \\
\hline 564. & $\begin{array}{l}\text { SW1/4 sec. 31, T. } 4 \text { S., R. } 6 \text { E. } \\
\text { North Branch Macon River at Ridge Road, } \\
\text { SE } 1 / 4 \text { sec. } 31, \text { T. } 4 \text { S., R. } 6 \text { E. }\end{array}$ & 13.5 & 13.7 & 6.9 & 716 & 817 & 14.6 \\
\hline 565. & $\begin{array}{l}\text { North Branch Macon River at Dennison Road, } \\
\text { SE } 1 / 4 \text { sec. } 5 \text {, T. } 5 \text { S., R. } 6 \text { E. }\end{array}$ & 15.5 & 10.9 & 9.7 & 699 & 817 & 12.1 \\
\hline 566. & $\begin{array}{l}\text { North Branch Macon River above unnamed } \\
\text { tributary, } \\
\text { SE1/4 sec. } 10, \text { T. } 5 \mathrm{~S} ., \text { R. } 6 \text { E. } \\
\text { Unnamed tributary at mouth, }\end{array}$ & 18.7 & 7.5 & 13.1 & 683 & 817 & 10.2 \\
\hline 568. & $\begin{array}{l}\mathrm{SE}^{1 / 4} \mathrm{sec} .10 \text {, T. } 5 \mathrm{~S} ., \mathrm{R} .6 \mathrm{E} \text {. } \\
\text { North Branch Macon River at unnamed } \\
\text { tributary, }\end{array}$ & 1.11 & 0 & 1.8 & 683 & 700 & 9.4 \\
\hline 569. & $\begin{array}{l}\mathrm{SE}^{1 / 4} \mathrm{sec} .10, \text { T. } 5 \text { S., R. } 6 \text { E. } \\
\text { North Branch Macon River at Highway } 23 \text {, }\end{array}$ & 19.8 & 7.5 & 13.1 & 683 & 817 & 10.2 \\
\hline 570. & $\begin{array}{l}\text { NE1/4 sec. } 26 \text {, T. } 5 \text { S., R. } 6 \text { E. } \\
\text { North Branch Macon River at Ostrander Road, }\end{array}$ & 22.2 & 3.9 & 16.7 & 662 & 817 & 9.3 \\
\hline 571. & $\begin{array}{l}\text { NW11/4 sec. } 25, \text { T. } 5 \text { S., R. } 6 \text { E. } \\
\text { North Branch Macon River above Bear Swamp } \\
\text { Creek, }\end{array}$ & 23.0 & 3.0 & 17.6 & 657 & 817 & 9.1 \\
\hline 572. & $\begin{array}{l}\text { NW11/4 sec. } 36, \text { T. } 5 \text { S., R. } 6 \text { E. } \\
\text { Bear Swamp Creek at North County Line } \\
\text { Road, }\end{array}$ & 23.7 & 2.0 & 18.6 & 652 & 817 & 8.9 \\
\hline 573. & $\begin{array}{l}\text { NE1/4 sec. } 24, \text { T. } 5 \text { S., R. } 5 \text { E. } \\
\text { Bear Swamp Creek above unnamed tributary, }\end{array}$ & 6.00 & 6.8 & 4.6 & 693 & 747 & 11.7 \\
\hline & NW1/4 sec. 29 , T. 5 S., R. 6.E. & 6.90 & 5.2 & 6.2 & 675 & 747 & 11.6 \\
\hline & NW11/4 sec. 29, T. 5 S., R. 6 E. & 1.39 & 0 & 2.3 & 675 & 705 & 13.0 \\
\hline 575. & $\begin{array}{l}\text { Bear Swamp Creek at unnamed tributary, } \\
\mathrm{NW}^{1} / 4 \text { sec. } 29, \text { T. } 5 \mathrm{~S} ., \text { R. } 6 \mathrm{E} \text {. }\end{array}$ & 8.29 & 5.2 & 6.2 & 675 & 747 & 11.6 \\
\hline 576. & $\begin{array}{l}\text { Bear Swamp Creek above Cone Drain, } \\
\text { NW11/4 sec. } 28 \text {, T. } 5 \text { S., R. } 6 \text { E. }\end{array}$ & 8.55 & 4.2 & 7.2 & 674 & 747 & 10.1 \\
\hline 578. & $\begin{array}{l}\text { Cone Drain at mouth, } \\
\text { NW1/4 sec. } 28, T \text {. } 5 \text { S., R. } 6 \text { E. } \\
\text { Bear Swamp Creek at Cone Drain, }\end{array}$ & 1.64 & 0 & 3.4 & 674 & 715 & 12.1 \\
\hline 579. & $\begin{array}{l}\text { NW1/4 sec. } 28 \text {, T. } 5 \text { S., R. } 6 \text { E. } \\
\text { Bear Swamp Creek above Center Creek, }\end{array}$ & 10.2 & 4.2 & 7.2 & 674 & 747 & 10.1 \\
\hline & & 12.0 & 1.8 & 9.6 & 665 & 747 & 8.5 \\
\hline
\end{tabular}


PHYSIOGRAPHIC DATA FOR SELECTED SUBBASINS IN THE RIVER RAISIN BASIN - Continue

\begin{tabular}{|c|c|c|c|c|c|c|c|}
\hline \multirow{2}{*}{$\begin{array}{c}\text { Num- } \\
\text { ber } \\
\text { key }\end{array}$} & Stream and location & \multirow{2}{*}{$\begin{array}{l}\text { Drainage } \\
\text { area } \\
(\mathrm{sq} \mathrm{mi})\end{array}$} & \multicolumn{2}{|c|}{ Miles } & \multicolumn{2}{|c|}{$\begin{array}{c}\text { Altitude } \\
\text { (ft above msl) }\end{array}$} & \multirow{2}{*}{$\begin{array}{l}\text { Average } \\
\text { slope } \\
\text { (ft per } \\
\text { mile) }\end{array}$} \\
\hline & 123456 Ranking order & & $\begin{array}{l}\text { above } \\
\text { mouth }\end{array}$ & $\begin{array}{l}\text { below } \\
\text { source }\end{array}$ & $\begin{array}{c}\text { at } \\
\text { site }\end{array}$ & $\begin{array}{c}\text { at } \\
\text { source }\end{array}$ & \\
\hline $\begin{array}{l}580 . \\
581 .\end{array}$ & $\begin{array}{l}\text { Center Creek above Rice Drain, } \\
\text { SE } 1 / 4 \mathrm{sec} .8, \text { T. } 5 \text { S., R. } 6 \text { E. } \\
\text { Rice Drain at mouth, }\end{array}$ & 1.98 & 4.2 & 2.9 & 695 & 730 & 13.0 \\
\hline 582. & $\begin{array}{l}\text { SE } 1 / 4 \text { sec. } 8, \text { T. } 5 \text { S., R. } 6 \text { E. } \\
\text { Center Creek at Rice Drain, }\end{array}$ & .42 & 0 & .8 & 695 & 711 & 20.0 \\
\hline 583. & $\begin{array}{l}\text { SE } 1 / 4 \text { sec. } 8, \text { T. } 5 \text { S., R. } 6 \text { E. } \\
\text { Center Creek above Nolan Engle Drain, }\end{array}$ & 2.40 & 4.2 & 2.9 & 695 & 730 & 12.1 \\
\hline 584. & $\begin{array}{l}\mathrm{SE}^{1 / 4} \mathrm{sec} .22, \mathrm{~T} .5 \mathrm{~S} ., \text { R. } 6 \text { E. } \\
\text { Nolan Engle Drain at mouth, }\end{array}$ & 4.48 & 1.0 & 6.1 & 669 & 730 & 10.0 \\
\hline 585. & $\begin{array}{l}\mathrm{SE}^{1 / 4} \mathrm{sec} .22 \text {, T. } 5 \mathrm{~S} ., \text { R. } 6 \text { E. } \\
\text { Center Creek at Nolan Engle Drain, }\end{array}$ & 1.12 & 0 & 2.0 & 669 & 690 & 10.5 \\
\hline 586. & $\begin{array}{l}\mathrm{SE}^{1} / 4 \text { sec. } 22 \text {, T. } 5 \text { S., R. } 6 \text { E. } \\
\text { Center Creek at mouth, }\end{array}$ & 5.60 & 1.0 & 6.1 & 669 & 730 & 10.0 \\
\hline 587. & $\begin{array}{l}\mathrm{NW} 1 / 4 \text { sec. } 26, \text { T. } 5 \text { S., R. } 6 \text { E. } \\
\text { Bear Swamp Creek at Center Creek, }\end{array}$ & 5.90 & 0 & 7.1 & 665 & 730 & 9.2 \\
\hline 588. & $\begin{array}{l}\text { NW1/4 sec. } 26, \text { T. } 5 \text { S., R. } 6 \text { E. } \\
\text { Bear Swamp Creek above Leet Weidner Drain, }\end{array}$ & 17.8 & 1.8 & 9.6 & 665 & 747 & 8.5 \\
\hline 589. & $\begin{array}{l}\mathrm{SEE}^{1 / 4} \mathrm{sec} .26, \mathrm{~T} .5 \mathrm{~S} ., \text { R. } 6 \mathrm{E} \text {. } \\
\text { Leet Weidner Drain at mouth, }\end{array}$ & 18.2 & .7 & 10.7 & 657 & 747 & 8.4 \\
\hline 590. & $\begin{array}{l}\text { SE } 1 / 4 \text { sec. } 26, \text { T. } 5 \text { S., R. } 6 \text { E. } \\
\text { Bear Swamp Creek at Leet Weidner Drain, }\end{array}$ & 2.08 & 0 & 2.4 & 657 & 675 & 7.5 \\
\hline 591. & $\begin{array}{l}\mathrm{SE}^{1 / 4} \mathrm{sec} .26, \mathrm{~T} .5 \mathrm{~S} ., \text { R. } 6 \text { E. } \\
\text { Bear Swamp Creek at mouth, }\end{array}$ & 20.2 & .7 & 10.7 & 657 & 747 & 8.4 \\
\hline 592. & 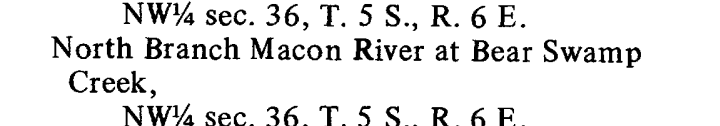 & 20.5 & 0 & 11.4 & 652 & 747 & 8.3 \\
\hline 593. & $\begin{array}{l}\text { NW1/4 sec. 36, T. } 5 \text { S., R. } 6 \text { E. } \\
\text { North Branch Macon River at Day Road, } \\
\text { SE1/4 sec. 36, T. } 5 \text { S.. R. } 6 \text { E. }\end{array}$ & 44.2 & 2.0 & 18.6 & 652 & 817 & 8.9 \\
\hline 594. & $\begin{array}{l}\text { North Branch Macon River at mouth, } \\
\text { SE } 1 / 4 \text { sec. 1, T. } 6 \text { S., R. } 6 \text { E. }\end{array}$ & 45.8 & 1.0 & 20.6 & 646 & 817 & 8.7 \\
\hline $\begin{array}{l}595 . \\
596 .\end{array}$ & $\begin{array}{l}\text { Macon River at North Branch Macon River, } \\
\text { SE } 1 / 4 \text { sec. } 1, \text { T. } 6 \text { S., R. } 6 \text { E. } \\
\text { Macon River at mouth, }\end{array}$ & 140 & 2.4 & 23.9 & 639 & 870 & 9.7 \\
\hline 597. & $\begin{array}{l}\text { NW1/4 sec. } 8, \text { T. } 6 \text { S., R. } 7 \text { E. } \\
\text { River Raisin at Macon River, }\end{array}$ & 144 & 0 & 26.3 & 634 & 870 & 9.0 \\
\hline 598. & $\begin{array}{l}\text { NW1/4 sec. 8, T. } 6 \text { S., R. } 7 \text { E. } \\
\text { River Raisin above Saline River, } \\
\text { SW11/4 Sec }\end{array}$ & 909 & 18.5 & 115.2 & 634 & 1,030 & 3.4 \\
\hline 599. & $\begin{array}{l}\text { SW1/4 sec. 9, T. } 6 \text { S., R. } 7 \text { E. } \\
\text { Saline River above Columbia Lake, }\end{array}$ & 909 & 17.5 & 116.2 & 633 & 1,030 & 3.4 \\
\hline 600. & $\begin{array}{l}\mathrm{NW} 1 / 4 \mathrm{sec} .3, \mathrm{~T} .4 \mathrm{~S} ., \text { R. } 4 \mathrm{E} \text {. } \\
\text { Unnamed tributary at mouth, }\end{array}$ & 3.56 & 44.5 & 2.0 & 860 & 980 & 60.0 \\
\hline 601. & $\begin{array}{l}\text { NW1/4 sec. 3, T. } 4 \text { S., R. } 4 \text { E. } \\
\text { Saline River at Austin Road, }\end{array}$ & 1.47 & 0 & 1.6 & 860 & 945 & 53.1 \\
\hline 602. & $\begin{array}{l}\mathrm{SW} 1 / 4 \mathrm{sec} .3, \mathrm{~T} .4 \mathrm{~S} ., \mathrm{R} .4 \mathrm{E} \text {. } \\
\text { Saline River above Joslin Lake outlet, }\end{array}$ & 7.24 & 43.3 & 3.2 & 853 & 980 & 39.7 \\
\hline 603. & $\begin{array}{l}\mathrm{SE}^{1 / 4} \mathrm{sec} .13, \mathrm{~T} .4 \mathrm{~S} ., \mathrm{R} .4 \mathrm{E} . \\
\text { Joslin Lake outlet at mouth, } \\
\mathrm{SE}^{1 / 4} \mathrm{sec} 13\end{array}$ & 13.2 & 38.7 & 7.8 & 817 & 980 & 20.9 \\
\hline 604. & $\begin{array}{l}\text { SE1/4 sec. 13, T. } 4 \text { S., R. } 4 \text { E. } \\
\text { Saline River at Joslin Lake outlet, }\end{array}$ & 1.69 & 0 & 2.3 & 817 & 849 & 13.9 \\
\hline 605 & $\begin{array}{l}\text { SE } 1 / 4 \text { sec. } 13, \mathrm{~T} .4 \mathrm{~S} ., \mathrm{R} .4 \mathrm{E} \text {. } \\
\text { Saline River above unnamed tributary, }\end{array}$ & 14.9 & 38.7 & 7.8 & 817 & 980 & 20.9 \\
\hline 606. & $\begin{array}{l}\mathrm{SW}^{1 / 4} \mathrm{sec} .18, \mathrm{~T} .4 \mathrm{~S} ., \mathrm{R} .5 \mathrm{E} \text {. } \\
\text { Unnamed tributary at mouth, }\end{array}$ & 15.0 & 38.3 & 8.2 & 814 & 980 & 20.2 \\
\hline 607. & $\begin{array}{l}\mathrm{SW} 1 / 4 \mathrm{sec} .18, \mathrm{~T} .4 \mathrm{~S} ., \mathrm{R} .5 \mathrm{E} \text {. } \\
\text { Saline River at unnamed tributary, }\end{array}$ & 5.26 & 0 & 3.3 & 814 & 834 & 6.1 \\
\hline 608 & $\begin{array}{l}\text { SW1/4 sec. } 18 \text {, T. } 4 \mathrm{~S} ., \text { R. } 5 \text { E. } \\
\text { Saline River above unnamed tributary, } \\
\mathrm{NW}^{1 / 4} \text { sec. } 17.4 \mathrm{~S} .5 \mathrm{E}\end{array}$ & 20.3 & 38.3 & 8.2 & 814 & 980 & 20.2 \\
\hline & NW11/4 sec. 17, T. 4 S., R. 5 E. & 22.5 & 37.0 & 9.5 & 808 & 980 & 18.1 \\
\hline
\end{tabular}


PHYSIOGRAPHIC DATA FOR SELECTED SUBBASINS IN THE RIVER RAISIN BASIN - Continu»d

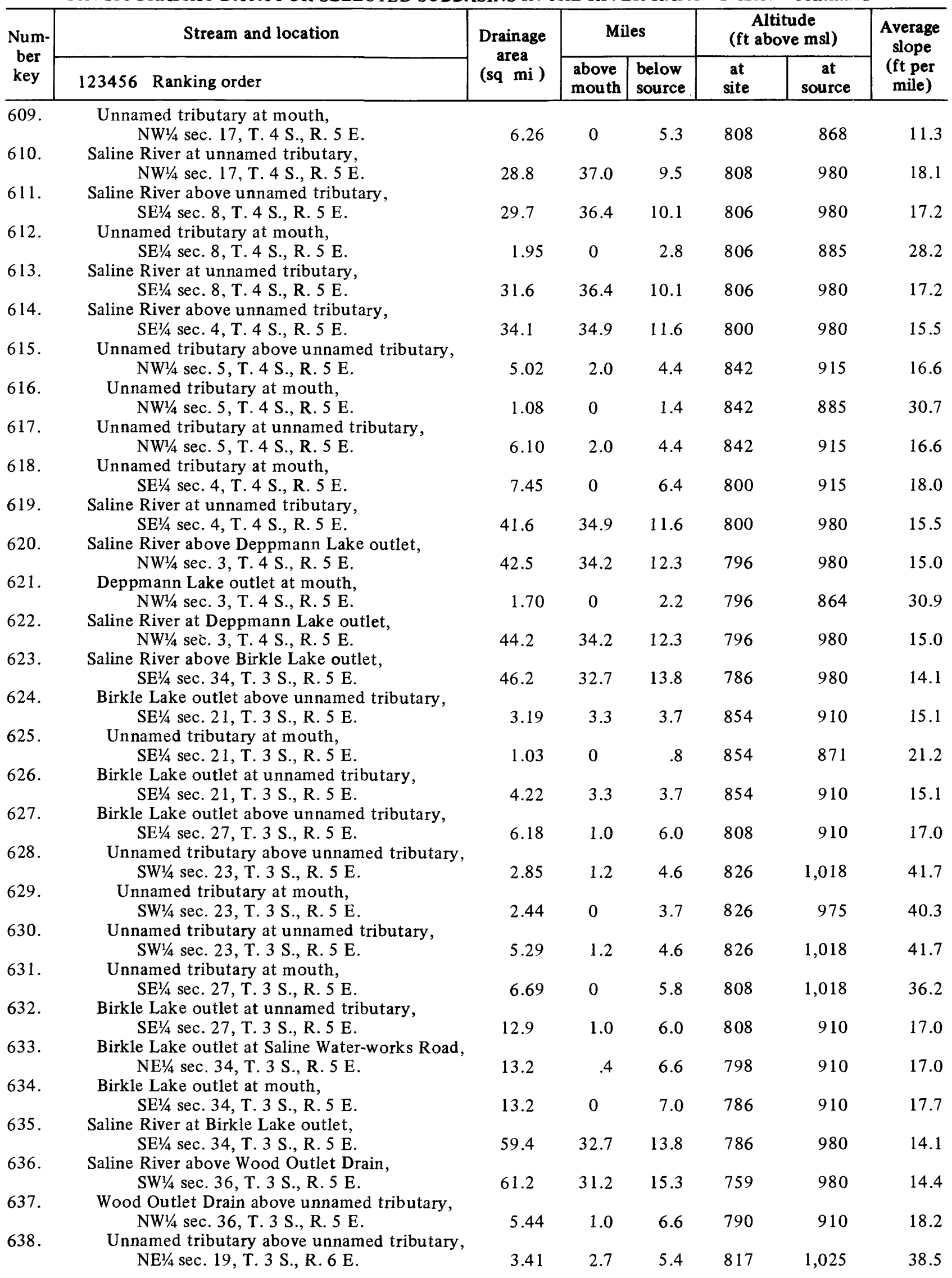


PHYSIOGRAPHIC DATA FOR SELECTED SUBBASINS IN THE RIVER RAISIN BASIN - Continued

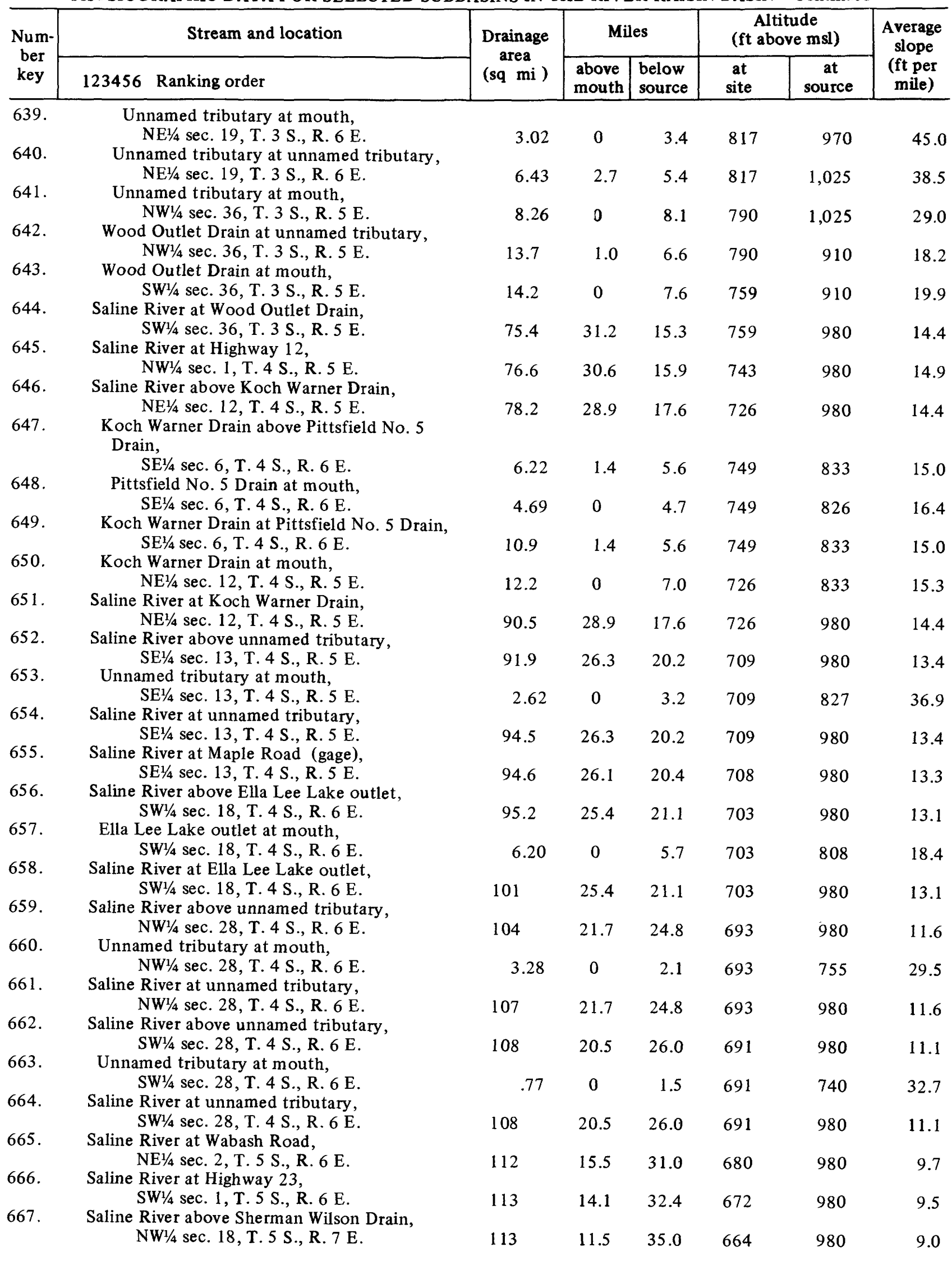


PHYSIOGRAPHIC DATA FOR SELECTED SUBBASINS IN THE RIVER RAISIN BASIN - Continued

\begin{tabular}{c|c}
\hline \multirow{2}{*}{$\begin{array}{r}\text { Num- } \\
\text { ber }\end{array}$} & Stream and location \\
\cline { 2 - 3 } key & $123456 \quad$ Ranking order \\
\hline
\end{tabular}

\begin{tabular}{|c|c|c|c|c|c|}
\hline \multirow{2}{*}{$\begin{array}{c}\text { Drainage } \\
\text { area } \\
\text { (sq } \mathrm{mi} \text { ) }\end{array}$} & \multicolumn{2}{|c|}{ Miles } & \multicolumn{2}{|c|}{$\begin{array}{c}\text { Altitude } \\
\text { (ft above msl) }\end{array}$} & \multirow{2}{*}{$\begin{array}{c}\text { Average } \\
\text { slope } \\
\text { (ft per } \\
\text { mile) }\end{array}$} \\
\hline & $\begin{array}{l}\text { above } \\
\text { mouth }\end{array}$ & $\begin{array}{l}\text { below } \\
\text { source }\end{array}$ & $\begin{array}{l}\text { at } \\
\text { site }\end{array}$ & $\begin{array}{c}\text { at } \\
\text { source }\end{array}$ & \\
\hline
\end{tabular}

668. Sherman Wilson Drain at mouth, NW $1 / 4$ sec. 18, T. 5 S., R. 7 E.

669. Saline River at Sherman Wilson Drain, NW $1 \frac{1}{4}$ sec. 18 , T. 5 S., R. 7 E.

670. Saline River above unnamed tributary, NW1/4 sec. 20 , T. 5 S., R. 7 E.

671. Unnamed tributary at mouth, NW $1 / 4$ sec. 20, T. 5 S.. R. 7 E.

672. Saline River at unnamed tributary, NW $1 / 4$ sec. 20 , T. 5 S., R. 7 E.

673. Saline River above Bear Creek, NE $1 / 4$ sec. 29 , T. 5 S., R. 7 E.

674. Bear Creek at mouth, NE $1 / 4$ sec. 29, T. 5 S., R. 7 E.

675. Saline River at Bear Creek, NE $1 / 4$ sec. 29 , T. 5 S., R. 7 E.

676. Saline River above Beaver Meadow Drain, $\mathrm{SE} 1 / 4$ sec. 32 , T. $5 \mathrm{~S}$., R. 7 E.

677. Beaver Meadow Drain at mouth, SE $1 / 4$ sec. 32, T. 5 S., R. 7 E.

678. Saline River at Beaver Meadow Drain, SE $1 / 4$ sec. 32 , T. 5 S., R. 7 E.

679. Saline River at Bigelow Road, NE $1 / 4$ sec. 8, T. 6 S., R. 7 E.

680. Saline River at mouth, SW $1 / 4$ sec. 9, T. 6 S., R. 7 E.

681. River Raisin at Saline River, SW $1 / 4$ sec. 9 , T. 6 S., R. 7 E.

682. River Raisin at Ida Maybee Road, Private claim 683, T. 6 S., R. 7 E.

683. River Raisin near Monroe (gage), Private claim 350, T. 6 S., R. 8 E.

684. River Raisin above Barnaby Drain, Private claim 446, T. 6 S., R. 8 E.

685. Barnaby Drain at mouth, Private claim 446, T. 6 S., R. 8 E.

686. River Raisin at Barnaby Drain, Private claim 446, T. 6 S., R. 8 E.

687. River Raisin above Brown Drain, Private claim 465, T. 6 S., R. 8 E.

688. Brown Drain at mouth, Private claim 465, T. 6 S., R. 8 E.

689. River Raisin at Brown Drain, Private claim 465, T. 6 S., R. 8 E.

690. River Raisin above Willow Run, Private claim 467, T. 6 S., R. 8 E.

691. Willow Run above Middle Branch Willow Run, Private claim 407, T. 6 S., R. 8 E.

692. Middle Branch Willow Run at mouth, Private claim 407, T. 6 S., R. 8 E.

693. Willow Run at Middle Branch Willow Run, Private claim 407, T. 6 S., R. 8 E.

694. Willow Run above North Branch Willow Run, Private claim 493, T. 6 S., R. 8 E.

695. North Branch Willow Run above Beitz Drain, Private claim 515, T. 6 S., R. 8 E.

696. Beitz Drain at mouth, Private claim 515, T. 6 S., R. 8 E.

697. North Branch Willow Run at Beitz Drain, Private claim 515, T. 6 S., R. 8 E.

\begin{tabular}{|c|c|c|c|c|c|}
\hline 1.33 & 0 & 2.6 & 664 & 690 & 10.0 \\
\hline 115 & 11.5 & 35.0 & 664 & 980 & 9.0 \\
\hline 116 & 8.4 & 38.1 & 654 & 980 & 8.6 \\
\hline 1.96 & 0 & 2.5 & 654 & 674 & 8.0 \\
\hline 118 & 8.4 & 38.1 & 654 & 980 & 8.6 \\
\hline 118 & 6.6 & 39.9 & 649 & 980 & 8.3 \\
\hline 3.96 & 0 & 5.0 & 649 & 680 & 6.2 \\
\hline 122 & 6.6 & 39.9 & 649 & 980 & 8.3 \\
\hline 124 & 3.5 & 43.0 & 639 & 980 & 7.9 \\
\hline 1.30 & 0 & 1.9 & 639 & 665 & 13.7 \\
\hline 126 & 3.5 & 43.0 & 639 & 980 & 7.9 \\
\hline 128 & .7 & 45.8 & 634 & 980 & 7.6 \\
\hline 128 & 0 & 46.5 & 633 & 980 & 7.5 \\
\hline 1,037 & 17.5 & 116.2 & 633 & 1,030 & 3.4 \\
\hline 1,042 & 13.3 & 120.4 & 623 & 1,030 & 3.4 \\
\hline 1,042 & 12.4 & 121.3 & 619 & 1,030 & 3.4 \\
\hline 1,043 & 11.3 & 122.4 & 613 & 1,030 & 3.4 \\
\hline 1.32 & 0 & 2.1 & 613 & 640 & 12.9 \\
\hline 1,044 & 11.3 & 122.4 & 613 & 1,030 & 3.4 \\
\hline 1,045 & 9.3 & 124.4 & 607 & 1,030 & 3.4 \\
\hline .57 & 0 & 1.5 & 607 & 622 & 10.0 \\
\hline 1,045 & 9.3 & 124.4 & 607 & 1,030 & 3.4 \\
\hline 1,046 & 7.7 & 126.0 & 603 & 1,030 & 3.4 \\
\hline 2.39 & 1.8 & 5.0 & 614 & 641 & 5.4 \\
\hline 1.50 & 0 & 3.0 & 614 & 633 & 6.3 \\
\hline 3.89 & 1.8 & 5.0 & 614 & 641 & 5.4 \\
\hline 4.06 & 1.5 & 5.3 & 611 & 641 & 5.7 \\
\hline 2.67 & 2.9 & 4.8 & 627 & 661 & 7.1 \\
\hline 1.52 & 0 & 2.7 & 627 & 641 & 5.1 \\
\hline 4.19 & 2.9 & 4.8 & 627 & 661 & 7.1 \\
\hline
\end{tabular}


PHYSIOGRAPHIC DATA FOR SELECTED SUBBASINS IN THE RIVER RAISIN BASIN -Continu?d

\begin{tabular}{|c|c|c|c|c|c|c|c|}
\hline \multirow{2}{*}{$\begin{array}{c}\text { Num- } \\
\text { ber } \\
\text { key }\end{array}$} & Stream and location & \multirow{2}{*}{$\begin{array}{c}\text { Drainage } \\
\text { area } \\
\text { (sq } \mathrm{mi} \text { ) }\end{array}$} & \multicolumn{2}{|c|}{ Miles } & \multicolumn{2}{|c|}{$\begin{array}{c}\text { Altitude } \\
\text { (ft above msl) }\end{array}$} & \multirow{2}{*}{$\begin{array}{c}\text { Average } \\
\text { slope } \\
\text { (ft per } \\
\text { mile) } \\
\end{array}$} \\
\hline & 123456 Ranking order & & $\begin{array}{l}\text { above } \\
\text { mouth }\end{array}$ & $\begin{array}{l}\text { below } \\
\text { source }\end{array}$ & $\begin{array}{l}\text { at } \\
\text { site }\end{array}$ & $\begin{array}{c}\text { at } \\
\text { source }\end{array}$ & \\
\hline \multirow{3}{*}{$\begin{array}{c}698 . \\
699 . \\
700 . \\
701 .\end{array}$} & $\begin{array}{l}\text { North Branch Willow Run at mouth, } \\
\text { Private claim } 493 \text {, T. } 6 \text { S., R. } 8 \text { E. } \\
\text { Willow Run at North Branch Willow Run, }\end{array}$ & 5.20 & $\mathbf{0}$ & 7.7 & 611 & 661 & \\
\hline & $\begin{array}{l}\text { Private claim } 493 \text {, T. } 6 \text { S., R. } 8 \text { E. } \\
\text { Willow Run at mouth, }\end{array}$ & 9.26 & 1.5 & 5.3 & 611 & 641 & 5.7 \\
\hline & $\begin{array}{l}\text { Private claim } 467 \text {, T. } 6 \text { S., R. } 8 \text { E. } \\
\text { River Raisin at Willow Run, }\end{array}$ & 10.1 & 0 & 6.8 & 603 & 641 & 5.6 \\
\hline \multirow{2}{*}{$\begin{array}{l}702 . \\
703 .\end{array}$} & $\begin{array}{l}\text { Private claim 467, T. } 6 \text { S., R. } 8 \text { E. } \\
\text { River Raisin above Brost Drain, }\end{array}$ & 1,056 & 7.7 & 126.0 & 603 & 1,030 & 3. \\
\hline & $\begin{array}{l}\mathrm{SW} 1 / 4 \text { sec. } 26, \mathrm{~T} .6 \mathrm{~S} ., \mathrm{R} .8 \mathrm{E} \text {. } \\
\text { Brost Drain at mouth, }\end{array}$ & 1,057 & 6.9 & 126.8 & 599 & 1,030 & 3. \\
\hline \multirow{2}{*}{$\begin{array}{l}704 . \\
705 .\end{array}$} & $\begin{array}{l}\mathrm{SW} 1 / 4 \text { sec. } 26 \text {, T. } 6 \mathrm{~S} \text {., R. } 8 \mathrm{E} \text {. } \\
\text { River Raisin at Brost Drain, }\end{array}$ & 1.04 & 0 & 2.9 & 599 & 624 & 8. \\
\hline & $\begin{array}{l}\text { SW1/4 sec. } 26 \text {, T. } 6 \text { S., R. } 8 \text { E. } \\
\text { River Raisin at dam, }\end{array}$ & 1,058 & 6.9 & 126.8 & 599 & 1,030 & 3. \\
\hline \multirow{3}{*}{$\begin{array}{l}706 . \\
707 .\end{array}$} & $\begin{array}{l}\text { Private claim 317, T. } 6 \text { S., R. } 8 \text { E. } \\
\text { River Raisin at Highway } 24\end{array}$ & 1,059 & 5.2 & 128.5 & 586 & 1,030 & \\
\hline & $\begin{array}{l}\text { Private claim 89, T. } 6 \text { S., R. } 9 \text { E. } \\
\text { Bates Drain at Highway 24, }\end{array}$ & 1,060 & 4.9 & 128.8 & 585 & 1,030 & 3. \\
\hline & $\begin{array}{l}\text { Private claim 89, T. } 6 \text { S., R. } 9 \text { E. } \\
\text { River Raisin at dam, }\end{array}$ & .90 & $\cdots-$ & 2.3 & 597 & 616 & 8. \\
\hline \multirow{2}{*}{$\begin{array}{l}708 . \\
709 .\end{array}$} & $\begin{array}{l}\text { Private claim 647, T. } 6 \text { S., R. } 9 \text { E. } \\
\text { River Raisin at dam, }\end{array}$ & 1,061 & 4.3 & 129.4 & 581 & 1,030 & \\
\hline & $\begin{array}{l}\text { Private claim 65, T. } 6 \text { S., R. } 9 \text { E. } \\
\text { River Raisin at dam, }\end{array}$ & 1,061 & 3.9 & 129.8 & 581 & 1,030 & 3.5 \\
\hline $\begin{array}{l}710 . \\
711 .\end{array}$ & $\begin{array}{l}\text { Private claim 353, T. } 6 \text { S., R. } 9 \text { E. } \\
\text { River Raisin at Highway 25, }\end{array}$ & 1,061 & 3.8 & 129.9 & 579 & 1,030 & \\
\hline \multirow{2}{*}{$\begin{array}{l}712 . \\
713 .\end{array}$} & $\begin{array}{l}\text { Private claim 353, T. } 7 \text { S., R. } 9 \text { E. } \\
\text { River Raisin at dam, }\end{array}$ & 1,061 & 3.7 & 130.0 & 579 & 1,030 & 3.5 \\
\hline & $\begin{array}{l}\text { Private claim 53, T. } 7 \text { S., R. } 9 \text { E. } \\
\text { River Raisin at dam (gage), }\end{array}$ & 1,062 & 3.6 & 130.1 & 579 & 1,030 & 3.5 \\
\hline \multirow{2}{*}{$\begin{array}{l}714 . \\
715 .\end{array}$} & $\begin{array}{l}\text { Private claim 159, T. } 7 \text { S., R. } 9 \text { E. } \\
\text { River Raisin at highway bridge, }\end{array}$ & 1,062 & 3.1 & 130.6 & 575 & 1,030 & \\
\hline & $\begin{array}{l}\text { Private claim 177, T. } 7 \text { S., R. } 9 \text { E. } \\
\text { River Raisin at Toledo Freeway, }\end{array}$ & 1,062 & 2.9 & 130.8 & 574 & 1,030 & 3.5 \\
\hline \multirow{2}{*}{$\begin{array}{l}716 . \\
717 .\end{array}$} & $\begin{array}{l}\text { Private claim 653, T. } 7 \text { S., R. } 9 \text { E. } \\
\text { River Raisin above Mason Run, }\end{array}$ & 1,063 & 2.0 & 131.7 & 573 & 1,030 & 3.5 \\
\hline & $\begin{array}{l}\text { NE1/4 sec. 9, T. } 7 \text { S., R. } 9 \text { E. } \\
\text { Mason Run above Karm Drain, }\end{array}$ & 1,063 & 1.3 & 132.4 & 572 & 1,030 & 3.5 \\
\hline \multirow{2}{*}{$\begin{array}{l}718 . \\
719 .\end{array}$} & $\begin{array}{l}\text { Private claim 480, T. } 6 \text { S., R. } 8 \text { E. } \\
\text { Karm Drain at mouth, }\end{array}$ & 1.12 & 8.0 & 4.2 & 617 & 635 & 4.3 \\
\hline & $\begin{array}{l}\text { Private claim 480, T. } 6 \text { S., R. } 8 \text { E. } \\
\text { Mason Run at Karm Drain, }\end{array}$ & .48 & 0 & 1.7 & 617 & 625 & 4.7 \\
\hline \multirow{2}{*}{$\begin{array}{l}720 . \\
721 .\end{array}$} & $\begin{array}{l}\text { Private claim 480, T. } 6 \mathrm{~S} ., \text { R. } 8 \mathrm{E} \text {. } \\
\text { Masun Run above Burdeau Drain, }\end{array}$ & 1.60 & 8.0 & 4.2 & 617 & 635 & 4.3 \\
\hline & $\begin{array}{l}\text { Private claim 732, T. } 6 \text { S., R. } 8 \text { E. } \\
\text { Burdeau Drain at mouth, }\end{array}$ & 1.74 & 7.5 & 4.7 & 614 & 635 & 4.5 \\
\hline \multirow{2}{*}{$\begin{array}{l}722 . \\
723 .\end{array}$} & $\begin{array}{l}\text { Private claim 732, T. } 6 \text { S., R. } 8 \text { E. } \\
\text { Mason Run at Burdeau Drain, }\end{array}$ & .44 & 0 & 1.9 & 614 & 625 & 5.8 \\
\hline & $\begin{array}{l}\text { Private claim 732, T. } 6 \text { S., R. } 8 \text { E. } \\
\text { Mason Run at Bates Road, }\end{array}$ & 2.18 & 7.5 & 4.7 & 614 & 635 & 4.5 \\
\hline \multirow{5}{*}{$\begin{array}{l}724 . \\
726 . \\
727 .\end{array}$} & $\begin{array}{l}\text { NW1/4 sec. } 24 \text {, T. } 6 \text { S., R. } 8 \text { E. } \\
\text { Moore Drain at mouth, }\end{array}$ & 2.55 & 6.5 & 5.7 & 609 & 635 & 4.6 \\
\hline & $\begin{array}{l}\text { Private claim 479, T. } 6 \text { S., R. } 8 \text { E. } \\
\text { Mason Run at Moore Drain, }\end{array}$ & .36 & 0 & 1.6 & 609 & 620 & 6.9 \\
\hline & $\begin{array}{l}\text { Private claim 479, T. } 6 \text { S., R. } 8 \text { E. } \\
\text { Mason Run above unnamed tributary, }\end{array}$ & 2.91 & 6.5 & 5.7 & 609 & 635 & 4.6 \\
\hline & $\begin{array}{l}\text { Private claim 486, T. } 6 \mathrm{~S} ., \text { R. } 8 \text { E. } \\
\text { Unnamed tributary at mouth, }\end{array}$ & 3.06 & 5.8 & 6.4 & 606 & 635 & 4.5 \\
\hline & Private claim 486, T. 6 S., R. 8 E. & .45 & 0 & 1.7 & 606 & 620 & 8.2 \\
\hline
\end{tabular}


PHYSIOGRAPHIC DATA FOR SELECTED SUBBASINS IN THE RIVER RAISIN BASIN - Continued

\begin{tabular}{|c|c|c|c|c|c|c|c|}
\hline \multirow{2}{*}{$\begin{array}{l}\text { Num- } \\
\text { ber } \\
\text { key }\end{array}$} & \multirow[t]{2}{*}{ Stream and location } & \multirow{2}{*}{$\begin{array}{l}\text { Drainage } \\
\text { area } \\
\text { (sq } \mathrm{mi})\end{array}$} & \multicolumn{2}{|c|}{ Miles } & \multicolumn{2}{|c|}{$\begin{array}{c}\text { Altitude } \\
\text { (ft above msl) }\end{array}$} & \multirow{2}{*}{$\begin{array}{l}\text { Average } \\
\text { slope } \\
\text { (ft per } \\
\text { mile) }\end{array}$} \\
\hline & & & $\begin{array}{l}\text { above } \\
\text { mouth }\end{array}$ & $\begin{array}{l}\text { below } \\
\text { source }\end{array}$ & $\begin{array}{l}\text { at } \\
\text { site }\end{array}$ & $\begin{array}{c}\text { at } \\
\text { source }\end{array}$ & \\
\hline 728. & $\begin{array}{l}\text { Mason Run at unnamed tributary, } \\
\text { Private claim } 486 \text {, T. } 6 \text { S., R. } 8 \text { E. } \\
\text { Mason Run at Telegraph Road, }\end{array}$ & 3.51 & 5.8 & 6.4 & 606 & 635 & 4.5 \\
\hline 730. & $\begin{array}{l}\text { Private claim } 89 \text {, T. } 6 \text { S., R. } 9 \text { E. } \\
\text { Mason Run at Monroe Street, }\end{array}$ & 4.20 & 4.4 & 7.8 & 597 & 635 & 4.9 \\
\hline 731. & $\begin{array}{l}\text { Private claim } 353 \text {, T. } 6 \text { S., R. } 9 \text { E. } \\
\text { Ives Drain at Monroe Street, }\end{array}$ & 4.75 & 3.5 & 8.7 & 591 & 635 & 5.1 \\
\hline 732. & $\begin{array}{l}\text { Private claim } 353 \text {, T. } 6 \text { S., R. } 9 \text { E. } \\
\text { Mason Run at Toledo Freeway, }\end{array}$ & 1.17 & $-\cdots$ & 2.0 & 595 & 605 & 5.0 \\
\hline 733. & $\begin{array}{l}\text { Private claim } 653 \text {, T. } 7 \text { S., R. } 9 \text { E. } \\
\text { Mason Run at mouth, }\end{array}$ & 7.37 & .9 & 11.3 & 575 & 635 & 5.3 \\
\hline 734. & $\begin{array}{l}\text { NE } 1 / 4 \text { sec. } 9, \text { T. } 7 \text { S., R. } 9 \text { E. } \\
\text { River Raisin at Mason Run, }\end{array}$ & 7.79 & 0 & 12.2 & 572 & 635 & 5.2 \\
\hline 735. & $\begin{array}{l}\text { NE1/4 sec. 9, T. } 7 \text { S., R. } 9 \text { E. } \\
\text { River Raisin at mouth, }\end{array}$ & 1,071 & 1.3 & 132.4 & 572 & 1,030 & 3.5 \\
\hline & SW1/4 sec. 11, T. 7 S., R. 9 E. & 1,072 & 0 & 133.7 & 572 & 1,030 & 3.4 \\
\hline
\end{tabular}


